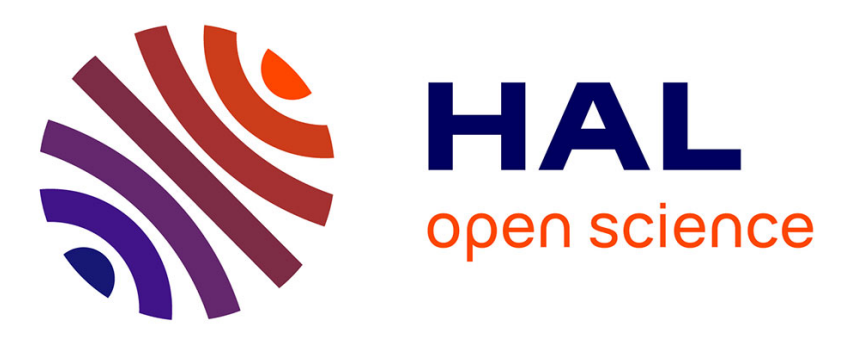

\title{
Analysis of self-broadened pure rotational and rovibrational lines of methyl chloride at room temperature
}

Cédric Bray, David Jacquemart, Nelly Lacome, Mickael Guinet, Arnaud Cuisset, Sophie Eliet, Francis Hindle, Gael Mouret, François Rohart, Jeanna Buldyreva

\section{To cite this version:}

Cédric Bray, David Jacquemart, Nelly Lacome, Mickael Guinet, Arnaud Cuisset, et al.. Analysis of self-broadened pure rotational and rovibrational lines of methyl chloride at room temperature. Journal of Quantitative Spectroscopy and Radiative Transfer, 2012, 116, pp.87-100. 10.1016/j.jqsrt.2012.09.013 . hal-00746031

\section{HAL Id: hal-00746031 \\ https://hal.sorbonne-universite.fr/hal-00746031}

Submitted on 29 Oct 2012

HAL is a multi-disciplinary open access archive for the deposit and dissemination of scientific research documents, whether they are published or not. The documents may come from teaching and research institutions in France or abroad, or from public or private research centers.
L'archive ouverte pluridisciplinaire HAL, est destinée au dépôt et à la diffusion de documents scientifiques de niveau recherche, publiés ou non, émanant des établissements d'enseignement et de recherche français ou étrangers, des laboratoires publics ou privés. 


\title{
Analysis of self-broadened pure rotational and rovibrational lines of methyl chloride at room temperature
}

\author{
C. Bray ${ }^{\text {a,b,** }}$, D. Jacquemart ${ }^{\text {a,b }}$, N. Lacome ${ }^{\text {a,b }}$,

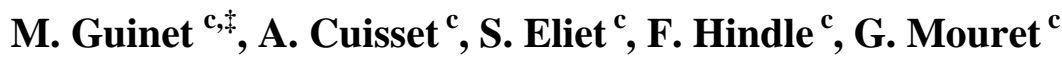 \\ F. Rohart ${ }^{\text {, }}$ J. Buldyreva ${ }^{\mathrm{e}}$
}

a UPMC Univ Paris 06, Laboratoire de Dynamique, Interactions et Réactivité, UMR 7075, Case Courrier 49, 4 Place Jussieu, 75252 Paris Cedex 05, France

b CNRS, UMR 7075, Laboratoire de Dynamique, Interactions et Réactivité, Case Courrier 49, 4 Place Jussieu, 75252 Paris Cedex 05, France

' Laboratoire de Physico-Chimie de l’Atmosphère, EA4493, Université du Littoral Côte d’Opale, 189A Avenue Maurice Schumann, 59140 Dunkerque, France

d Laboratoire de Physique des Lasers, Atomes et Molécules, UMR-CNRS 8523, Université de Lille 1, 59655 Villeneuve d’Ascq Cedex, France

e Institut UTINAM, UMR CNRS 6213, Université de Franche-Comté, 16 Route de Gray, 5030 Besançon cedex, France

${ }^{\ddagger}$ Presently in UPMC Univ Paris 06, Laboratoire de Dynamique, Interactions et Réactivité, UMR 7075, Case Courrier 49, 4 Place Jussieu, 75252 Paris Cedex 05, France

Number of Figures: 10

Number of Tables: 4

*Corresponding author: Bray Cédric (Email: bray@spmol.jussieu.fr), Tel: 0144273682

\section{Keywords:}

Methyl chloride; Self-broadening; Rotational dependences; Infrared; Terahertz; Semiclassical calculation. 


\begin{abstract}
Rovibrational absorption spectra of methyl chloride in the spectral region between 2800 and $3200 \mathrm{~cm}^{-1}$ were recorded with a high-resolution Fourier spectrometer. A multispectrum fitting procedure was used to analyze 527 transitions of the $v_{1}$ band and to retrieve the selfbroadening coefficients for various $J$ - and $K$-values with an estimated accuracy around $8 \%$. Pure rotational transitions of $\mathrm{CH}_{3} \mathrm{Cl}$ in the submillimeter/terahertz region $(0.2-1.4 \mathrm{THz})$ were also investigated using two complementary techniques of frequency-multiplication and continuous-wave photomixing. 43 pure rotational self-broadening coefficients were extracted with the accuracy between 3 and 5\%. The whole set of measured values was used to model the $J$ - and $K$-rotational dependences of the self-broadening coefficients by second-order polynomials. In addition, semi-classical calculations were performed, based on the real symmetric-top geometry of the active molecule, an intermolecular potential model including not only the dominant electrostatic but also the short-range forces, as well as on an exact classical treatment of the relative translational motion of the colliding partners. Comparison of all experimental and theoretical results shows similar rotational dependences and no significant vibrational dependence, so that extrapolations to other spectral regions should be straightforward.
\end{abstract}




\section{Introduction}

Methyl chloride is one of the most abundant organohalogen, anthropogenic or natural in the Earth's atmosphere, so that its quantification and monitoring are of great importance for atmospheric studies [1-4]. It has a rather strong signature around $3000 \mathrm{~cm}^{-1}$ ( $v_{1}$ band region) which was recently used by the Atmospheric Chemistry Experiment (ACE) satellite mission to produce the first global distribution of methyl chloride in the upper troposphere and stratosphere [5]. Prior to a precise quantification of $\mathrm{CH}_{3} \mathrm{Cl}$ in the atmosphere, the accurate determination of line profile parameters of $\mathrm{CH}_{3} \mathrm{Cl}$ is required. These parameters have to be determined from laboratory experiments. For the symmetric top $\mathrm{CH}_{3} \mathrm{Cl}$, numerous measurements have to be performed in order to reach transitions with various $J$ and $K$ values and obtain the rotational $J$ - and $K$-dependences of line broadening coefficients.

Self-broadening coefficients of methyl chloride infrared transitions have been previously studied experimentally for the $v_{2}, 2 v_{3}$ and $v_{5}$ bands [6] as well as for the $v_{3}$ band [7-9]. In Ref. [6], Chackerian et al. recorded high-resolution Fourier Transform spectra and retrieved 423 self-broadening coefficients exhibiting well-pronounced dependences on the quantum number $J$, but no dependence on the quantum number $K$. In Refs. [7-9] a diode laser spectrometer characterized by a high signal-to-noise ratio but limited to a narrow spectral range was used to obtain 29 self-broadening coefficients. In Ref. [9] semi-classical calculations were also performed to evaluate the $J$-and $K$-dependences of self-broadening $\mathrm{CH}_{3} \mathrm{Cl}$ coefficients at 200 and $296 \mathrm{~K}$ but a strong over-estimation of calculated line widths was stated. The determination of self-broadening coefficients of $\mathrm{CH}_{3} \mathrm{Cl}$ lines from pure rotational transitions using electronic sources was limited to old studies focused on the lowest energy $(J=0-1)$ transition [10]. Using far infrared lasers, some measurements [11] were performed on isolated terahertz (THz) lines but without any possibility to study the $J$ and $K$ rotational dependences. More recently, Harde et al. [12] via THz coherent transients and THz time-domain spectroscopy were able to study the $J$ dependence of collisional-broadened $\mathrm{CH}_{3} \mathrm{Cl}$ line-widths. Limited by the resolution of the spectrometer, the $J$ dependence was retrieved with measurements at pressures higher than 75 Torr where strong nonlinear broadenings are observed.

This work is dedicated to the study of room-temperature $\mathrm{CH}_{3} \mathrm{Cl}$ self-broadening coefficients in different spectral regions and by various experimental techniques. Self- 
broadening coefficients have been measured in the "Laboratoire de Dynamique, Interactions et Réactivité" (LADIR) for transitions of $v_{1}$ band around $3000 \mathrm{~cm}^{-1}$ from the analysis of infrared Fourier-transform (FT) spectra. Self-broadening coefficients of pure rotational transitions have also been measured in the "Laboratoire de Physico-Chimie de l'Atmosphère” (LPCA) in terahertz region using a continuous-wave photomixing spectrometer and in the “Laboratoire de Physique des Lasers, Atomes et Molécules” (PhLAM) using a frequencymodulation spectrometer. The observed $J$-and $K$-rotational dependences are also modeled by a second-order polynomial with empirical coefficients deduced from fitting to the bulk of obtained experimental data. In addition, semi-classical calculations of self-broadening coefficients are performed in order to compare with the empirical model but mainly to be able to calculate self-broadening coefficients for transitions with high $K$ and $J$ values that have not been observed.

In the next section the experimental conditions and brief descriptions of the instrumental setups used in the present work are presented. The retrieval of self-broadening coefficients is described in Section 3. The modeling of the measurements by an empirical polynomial function and the details of the semi-classical calculations are presented in Sections 4 and 5, respectively. Section 6 is dedicated to comparisons of our results with those available in the literature. The final section outlines the general conclusions and some perspectives of the present study.

\section{Experimental conditions}

\subsection{Infrared Fourier transform spectrometer}

Fourier transform spectra have been recorded between 2800 and $3200 \mathrm{~cm}^{-1}$ using the rapid scan Bruker IFS 120 HR interferometer of the LADIR. The experimental conditions are summarized in Table 1. This set of spectra have been already analyzed [13] with a multispectrum fitting procedure to measure line positions and intensities of around 500 transitions in the $3.4 \mu \mathrm{m}$ spectral region. More details concerning the experimental setup and conditions can be found in Ref. [13].

\section{$2.2 \mathrm{THz}$ photo-mixing spectrometer}


A photomixing $\mathrm{CW}-\mathrm{THz}$ spectrometer was also used at $\mathrm{LPCA}$ to record $\mathrm{CH}_{3} \mathrm{Cl}$ spectra between 0.8 and $1.4 \mathrm{THz}$ corresponding to $31 \leq J \leq 50$. This spectrometer is based on an ultra fast photomixer converting a beating between two near infrared laser diodes into the $\mathrm{THz}$ domain. This opto-electronic frequency-conversion provides a $\mathrm{THz}$ radiation at the frequency difference between the two infrared sources keeping their spectral purities. The diodes were locked onto a frequency comb generated from a femtosecond laser itself locked onto a frequency standard (provide by a GPS). The synthesized THz source exhibits metrological characteristics (a relative frequency accuracy of $10^{-8}$ and an apparatus function in the order of $30 \mathrm{kHz}$ FWHM). More details can be found in [14,15]. Different stainless steel absorption cells, with various lengths $(25,65,85,120 \mathrm{~cm})$, equipped with PTFE windows were used depending on the line intensity (see Table 1). For $J=31$, a room temperature Schottky diode detector (VDI). For the other values of $J$, the detection has been used with a liquid He-cooled bolometer (QMC Instruments). The spectra were recorded at room temperature (293 $\pm 1 \mathrm{~K})$. The pressure was measured with a 1 mbar full scale pressure gauge (Alcatel ADS-1004) with a stated accuracy of $0.25 \%$. A total of $130 \mathrm{cw}-\mathrm{THz}$ spectra in a $75-1000 \mathrm{mTorr}$ pressure range were recorded. More details on the experiments are available in Refs. $[16,17]$ dealing with the $\mathrm{O}_{2}$ and $\mathrm{N}_{2}$ broadening coefficient measurements of $\mathrm{CH}_{3} \mathrm{Cl}$.

\subsection{Millimeter frequency-modulation spectrometer}

The measurements on the $J=6 \rightarrow 7$ rotational line of ${ }^{12} \mathrm{CH}_{3}{ }^{35} \mathrm{Cl}$ at $186 \mathrm{GHz}$ were performed with the PhLAM frequency-modulation spectrometer that has been described in details in previous papers $[17,18]$, so we only recall its principal features. The electromagnetic (e.m.) source consisted of a synthesizer (Agilent E8257D) locked onto a GPS receiver and followed by an active sextupler (Spacek Inc.). The e.m. power was detected by an InSb liquid He-cooled bolometer (QMC Instruments) and the corresponding signal, demodulated by a lock-in amplifier (Ametek 7270DSP) operating at twice the modulation frequency $(f=10.5$ $\mathrm{kHz}$ ), was stored in a computer which managed all the spectrometer.

The absorption cell was a stainless tube having a $1.1 \mathrm{~m}$ length and a $4 \mathrm{~cm}$ diameter and set at room temperature. Gas pressure was measured with a 1-Torr capacitance manometer (MKS Baratron 627A) having a 0.1 mTorr resolution and a stated reading accuracy of $0.12 \%$. The $\mathrm{CH}_{3} \mathrm{Cl}$ sample (Aldrich) in natural abundance had a commercial purity better than $99.9 \%$ and was used without further purification. The experimental conditions are summarized in Table 1. 


\section{Measurements of self-broadening coefficients}

Measurements of ${ }^{12} \mathrm{CH}_{3} \mathrm{Cl}$ transitions have been obtained in the pure rotational region and for the $v_{1}$ band around $3.4 \mu \mathrm{m}$. The hyperfine structure was taken into account for pure rotational transitions but not in to the IR region where it could be neglected. Because of the nuclear spin $I=3 / 2$ of the ${ }^{35} \mathrm{Cl}$ or ${ }^{37} \mathrm{Cl}$ atom, rotational levels are split due the interaction of the chlorine quadrupole moment and the molecular field gradient. Each $K$-component is split into 12 quadrupolar components, at least for rotational lines considered in this work that involve sufficiently large $J$-values. Defining the total angular momentum $\vec{F}$ as $\vec{F}=\vec{J}+\vec{I}$, the four main components related to $\Delta F=+1$ are of comparable intensities, about $1 / 4$ of the unperturbed line intensity, and are better resolved as $J$ is smaller and $K$ is higher. In contrast, $\Delta F=0$ component intensities are quite lower and become much lesser as $J$ gets larger whereas the $\Delta F=-1$ components are always practically negligible. In the pure rotational band case, this hyperfine structure has already been studied in details [19], and was taken into account in photomixing and frequency-modulation measurements of this work. Furthermore and following our previous studies on the $\mathrm{CH}_{3} \mathrm{Cl}$ relaxation induced by $\mathrm{N}_{2}$ and $\mathrm{O}_{2}[16,17]$ or according to our conclusions on the $\mathrm{H}_{2}$-induced relaxation [18, see also 20,21], all hyperfine components of a given $(J, K)$ line were considered as having the same collisional broadening. In the $v_{1}$ rovibrational band case, it can be assumed the total frequency spreading of the quadrupolar structure related to $\Delta F=+1$ components is comparable to that observed for the pure rotational band, that is a $\approx 1.10^{-3} \mathrm{~cm}^{-1}$ maximum value observed for the $J=K=2$ line, and much lesser for higher $J$-values [19]. Consequently, taking into account of the large Doppler effect $\left(\approx 3.10^{-3} \mathrm{~cm}^{-1} \mathrm{HWHM}\right.$ ), rovibrational $(J, K)$ lines were considered as single lines. Note that in the following of the text, figures and tables, $J$ and $K$ refer to the quantum numbers associated to the lower state of the transition.

\subsection{Infrared measurements with the Fourier transform spectrometer}

In the IR spectral region, measurements have been performed for 527 lines with $J$ and $K$ values ranging from 0 up to 42 and from 0 up to 11, respectively. A multispectrum fitting procedure [22] has been used to adjust the line profiles of transitions simultaneously on the various experimental spectra of Table 1. Measurements of line positions and intensities have 
already been presented in Ref. [13]. For all spectra, a Voigt profile was used and no deviation from this profile has been observed in the residuals of the fits as shown in Fig. 1 of Ref. [13]. More details concerning the fitting procedure applied for these spectra can be found in Ref. [13]. The self-broadening coefficients were measured with an estimated accuracy around 8\%, assuming the effects of the collisions similar for $\mathrm{CH}_{3}{ }^{35} \mathrm{Cl}$ and $\mathrm{CH}_{3}{ }^{37} \mathrm{Cl}$ :

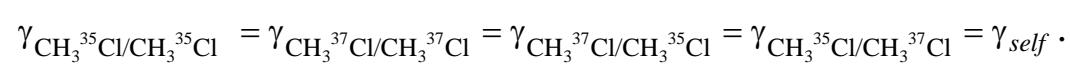

No temperature correction has been done since temperatures of all spectra are close to room temperature.

The whole set of 527 experimental self-broadening coefficients is given as supplementary material and a sample of these measurements is given in Table 2. These selfbroadening coefficients have been used to adjust an empirical model described in Section 4.1.

\subsection{THz measurements with the photo-mixing spectrometer}

Transitions of the most abundant $\mathrm{CH}_{3}{ }^{35} \mathrm{Cl}$ isotopologue have been studied for $J$-values ranging from 31 up to 50 and for $K$-values up to 12 . An example of three $K$ components of the $J=40$ line recorded around $36.18 \mathrm{~cm}^{-1}$ region $(1.087 \mathrm{THz})$ at four different pressures is presented in Fig. 1. Each set of measurements consisted of the use of 5-6 experimental spectra recorded at different pressures that were simultaneously adjusted with a similar multispectrum fitting procedure [23]. As explained previously, the $\mathrm{CH}_{3} \mathrm{Cl}$ hyperfine structure was taken into account in the submillimeter $/ \mathrm{THz}$ spectra to accurately retrieve the self broadening coefficients [16,17,24]. For each $(J, K)$ line, we have considered the four most intense hyperfine components $\Delta F=+1$; the other hyperfine components with $\Delta F=0$ and -1 with weaker intensities have been ignored. The lines were then modeled with a sum of four Voigt profiles with the Gaussian line-width fixed to the Doppler value and the Lorentzian line-width fitted to the profile. As quoted before, we have assumed identical self-broadenings for the four $\Delta F=+1$ components of a given $K$-value. Dealing with the intensities, only the strongest hyperfine component was adjusted, the intensities of the three weaker lines being fixed to the HITRAN2008 values [25]. The frequencies were adjusted constraining the three frequency differences to the JPL data [19]. Lastly, the observed spectrum was fitted introducing the Beer-Lambert law and the base line was approximated with a second order polynomial. As a result, signal to noise ratios between 40 and 90 were obtained. The standard deviation of the retrieved self-broadening parameters was calculated to $2.5 \%$ of broadenings for the $K=0-6$ 
components and $4.3 \%$ for $K=7,9$ and 12 components. Finally, the accuracy of these measurements takes into account the measurement precision and the possible biases (pressure gauge accuracy $(1 \%)$, gas purity $(0.5 \%)$, gas contamination $(0.3 \%)$, temperature variation (0.3\%), zero transmission determination $(0.1 \%)$ ), quadratically added; all the details concerning the evaluation of the systematic errors are given in [16]. We estimated the accuracy to $3 \%$ for the self-broadening coefficients of the $K=0-6$ components and to $5 \%$ for the $K=7,9$ and 12 ones. The results are presented in Table 3 .

\subsection{Millimeter measurements with the frequency-modulation spectrometer}

Due to the large intensity of $\mathrm{CH}_{3}{ }^{35} \mathrm{Cl}$ lines in the pure rotational region [19] and taking account of the cell length, the self broadening could be studied with the frequency-modulation technique for the $J=6 \rightarrow 7$ transition at $186 \mathrm{GHz}$ only. As an example, the corresponding absorption spectrum recorded at a pressure of 19.9 mTorr is presented in Fig. 2. In addition to the second derivative appearance of line shapes due the frequency modulation technique, this spectrum is quite complex because of the hyperfine structure due to the ${ }^{35} \mathrm{Cl}$ atom. Each $K$-component is split into 4 main quadrupolar hyperfine components corresponding to $\Delta F=$ +1 , and smaller ones corresponding to $\Delta F=0$, the $\Delta F=-1$ components having a negligible intensity. Optical thicknesses of main hyperfine components being as large as about 0.18-0.25 for $K=0-3$ and $0.06-0.11$ for $K=4-6$, the optically thin sample approximation is not valid and the Beer-Lambert law had to be considered. This was done with line intensities fixed at their theoretical values obtained from Ref. [19] since the frequency modulation technique did not allowed an absolute determination of the experimental absorption. In this purpose, observed spectra were fitted using an extension of the code used in Ref. [18]. In a first step, the total absorbance $A(v)$ was computed as the sum of the absorbance contributions $a_{j}(v)$ corresponding to the different hyperfine $j$-components, which allowed for a determination of the sample transmission $T(v)=\exp [-A(v)]$. In a second step, the signal $S(v)$ detected with the $2 f$-frequency modulation technique used was obtained from a generalization of Dore method $[18,26]$. More precisely, let us define the sample correlation function $\Theta(\tau)$, that is the time domain response of the sample after a pulse excitation taking place at time $\tau=0$, which is given by the Fourier transform of $T(v)$. Then, the detected signal $S(v)$ is proportional to the real part of a Fourier transform written as in Ref. [18]: 


$$
S(v) \propto \operatorname{Re}\left\{\int_{0}^{\infty} \Theta(\tau) \mathrm{J}_{2}\left[2 \pi m \tau \operatorname{sinc}\left(2 \pi v_{m} \tau / 2\right)\right] \cos \left(2 \pi v_{m} \tau\right) \exp (-2 \pi i v \tau) \mathrm{d} \tau\right\},
$$

where $\mathrm{J}_{2}[x]$ stands for the second-order Bessel function, $m$ and $v_{m}$ represent the modulation depth and frequency, respectively, and $\operatorname{sinc}(x)$ means $\sin (x) / x$.

According to above discussions, experimental spectra have been considered as a superposition of independent Voigt profiles with a same relaxation rate for a given $K$-value. Line amplitudes were fixed at their theoretical values whereas their frequencies were left adjustable in so far as the spectral resolution was sufficient. Finally, Doppler broadening as well as modulation depth and frequency were fixed at values corresponding to experimental conditions and, as usual, fits included adjustments of a scaling factor and a linear base line.

Figure 2 shows the result of a fit performed on the $J=6 \rightarrow 7$ transition at $186 \mathrm{GHz}$. A rather good agreement is obtained for the whole spectrum, namely for the $K=4-6$ components. From 5 records with pressures ranging from 20 up to 100 mTorr, the linear dependence on pressure of the collisional broadening of each $K$-component has been analyzed: corresponding results are reported in Table 3.

Although it is out the scope of the present paper, it is worth noting that the $K=5$ and 6 components exhibit clear departures from the Voigt profile characteristic of line narrowing effects [27]. According to the large values of corresponding broadening parameters (from 310 up to $480 \times 10^{-3} \mathrm{~cm}^{-1}$.atm ${ }^{-1}$, that is at least 10 times larger than the kinetic diffusion parameter $\beta_{\text {kin }} \cong 3310^{-3} \mathrm{~cm}^{-1}$.atm ${ }^{-1}$ ), it can be claimed [27-29] that Dicke effect plays no role but that these features result nearly exclusively from the speed dependence of relaxation rates. However, such a study could not been done in details because of an insufficient signal to noise ratio.

\section{Empirical modeling of experimental measurements}

The large scale of measurements coming from various experimental setups is coherent and shows no vibrational dependence and similar rotational dependences (see Figs. 3-5). The whole set of measurements presented in Section 3 (see Table 3 and supplementary data) has been used to generate empirical coefficients that allow to reproduce the experimental trends of the $J$ and $K$ rotational dependences. This extensive set of measured self-broadening coefficients, obtained for values of $K$ from 0 to 12 and for $J$ values from 0 to 50 allowed the accurate study of the rotational $J$ and $K$ dependences. Since no systematic discrepancy has 
been observed for self-broadening coefficients of the various sub-branches, all the measurements have been analyzed together taking into account only the rotational quantum numbers $J$ and $K$ of the lower state of the transitions.

This empirical model was previously used in works on $\mathrm{CH}_{3} \mathrm{Br}$ [30] and $\mathrm{CH}_{3} \mathrm{Cl}$ [31] to describe the $K$-dependence for transitions having same the $J$ value. Each set of broadening coefficients characterized by the same $J$ value is fitted with a polynomial expansion of the second order in $K$ (fixing the first-order term to zero).

$$
\gamma_{J}(K)=a_{J}^{0}+a_{J}^{2} K^{2} .
$$

This model is then applied for all $J$ values measured. Example of the fit of the $K$ dependence for $J=6,10,23,28,31$ and 50 transitions is given in Fig. 3. The two coefficients $a_{J}^{0}$ and $a_{J}^{2}$ obtained for each set of same value of $J$ have then been plotted versus $J$ in Fig. 4, and are given together with their smoothed values in Table 4. The use of THz and millimeter measurements together with IR measurements allowed to improve the model especially for high $J$ values.

\section{Semi-classical calculations}

The quite high value of the $\mathrm{CH}_{3} \mathrm{Cl}$ dipole moment leads to strong long-range interactions between the active and perturbing molecules. For such molecular systems the usual line-width expression [32]

$$
\gamma_{f i}=\frac{n \overline{\mathrm{V}}}{2 \pi c} \int_{0}^{\infty} 2 \pi b d b\left(1-\mathrm{e}^{-\operatorname{Re} S_{2}}\right)
$$

( $n$ is the number density of perturbing particles, $\overline{\mathrm{v}}$ is the mean thermal velocity, $b$ is the impact parameter further replaced by the distance of the closest approach $r_{c}, S_{2}$ are the second-order contributions to the scattering matrix) and the modified formula [33]

$$
\gamma_{f i}=\frac{n \overline{\mathrm{v}}}{2 \pi c} \int_{0}^{\infty} 2 \pi b d b\left(1-\mathrm{e}^{-\mathrm{Re}\left\langle S_{2}\right\rangle_{J_{2}}}\right)
$$

(performing the average $\langle\ldots\rangle_{J 2}$ on the perturber's rotational states $J_{2}$ as the cumulant average in order to insure a correct application of the cumulant expansion) are expected to give noticeably different results [34]. In order to check this point, we have used both Eqs (4) and (5) in our calculations. 
When the exact-trajectory model [35] is chosen to describe the relative translational motion in the field of an isotropic potential, the theoretical expressions of the real parts of the $S_{2}$ terms for two symmetric tops of $\mathrm{CH}_{3} \mathrm{X}$-type can be either deduced from the general formulae [36] for two asymmetric colliders or calculated directly from the rotationally invariant representation of the intermolecular interaction potential

$$
V(1,2, \vec{r})=\sum_{\substack{l_{1} l_{2} l \\ k_{1} k_{2}}} V_{l_{1} l_{2} l}^{k_{1} k_{2}}(r) \sum_{m_{1} m_{2} m} C_{l_{1} m_{1} l_{2} m_{2}}^{l m} D_{m_{1} k_{1}}^{l_{1}}{ }^{*}\left(\varphi_{1}, \theta_{1}, \chi_{1}\right) D_{m_{2} k_{2}}^{l_{2}}\left(\varphi_{2}, \theta_{2}, \chi_{2}\right) C_{l m}^{*}(\theta, \varphi) .
$$

Here the product $D_{m_{1} k_{1}}^{l_{1}}{ }^{*}\left(\varphi_{1}, \theta_{1}, \chi_{1}\right) D_{m_{2} k_{2}}^{l_{2}}{ }^{*}\left(\varphi_{2}, \theta_{2}, \chi_{2}\right) C_{l m}^{*}(\theta, \varphi)$ stands for the rotationally invariant basis in the laboratory-fixed frame (asterisks mark the complex conjugation), $C_{l_{1} m_{1} l_{2} m_{2}}^{l m}$ are the Clebsch-Gordan coefficients and the radial potential components $V_{l_{1} l_{2} l}^{k_{1} k_{2}}(r)$ contain contributions from various kinds of intermolecular interactions (electrostatic, induction, dispersion, short-range repulsion). In the present work we have considered $l_{1} \leq 2$ and $l_{2} \leq 2$ and composed the potential as a sum of electrostatic $(e)$ and pairwise atom-atom ( $a$ a) interactions. For the electrostatic terms we have included the leading dipole-dipole, the dipole-quadrupole and the quadrupole-quadrupole terms for which $k_{1}=k_{2}=0$ : $V^{00 e}=V_{112}^{00 e}+V_{123}^{00 e}+V_{213}^{00 e}+V_{224}^{00 e}$ (with the numerical values $\mu=1.89592 \mathrm{D}$ for the dipole moment and $Q=1.23 \mathrm{D} \AA$ for the quadrupole moment of $\mathrm{CH}_{3} \mathrm{Cl}$ [37]). For the atomatom terms the radial potential components are determined by the interatomic parameters $d_{i j}$ and $e_{i j}$ (which are connected to the Lennard-Jones parameters $\varepsilon_{i j}, \sigma_{i j}$ of the atoms $i$ and $j$ $\left.\operatorname{via} d_{i j}=4 \varepsilon_{i j} \sigma_{i j}^{12}, e_{i j}=4 \varepsilon_{i j} \sigma_{i j}^{6}\right)$ and by the orientations of each atom $\Omega_{1 i / 2 j}^{\prime}$ in the corresponding molecular frame:

$$
V_{l_{1} l_{2} l}^{k_{1} k_{2} a-a}(r)=\sum_{i, j}\left[d_{i j} f_{l_{1} l_{2} l}^{12}\left(r_{1 i}, r_{2 j}, r\right)-e_{i j} f_{l_{1} l_{2} l}^{6}\left(r_{1 i}, r_{2 j}, r\right)\right] Y_{l_{1} k_{1}}\left(\Omega_{1 i}^{\prime}\right) Y_{l_{2} k_{2}}\left(\Omega_{2 j}^{\prime}\right)(7)
$$

(explicit forms of the functions $f_{l_{1} l_{2} l}^{6}, f_{l_{1} l_{2} l}^{12}$ coming from the two-center expansion of the interatomic distances in the laboratory-fixed frame can be found, for instance, in Ref. [16]). With the anisotropic potential of Eq. (6), the second-order contribution composed of three terms $S_{2, i 2}, S_{2, f 2}, S_{2, f 2 i 2}$ is given by

$$
S_{2, i 2}=\frac{2 r_{c}^{2}}{\hbar^{2} \bar{v}^{2}} \sum_{\substack{l_{1} l_{2} l \\ k_{1} k_{2}}}\left[\left(2 l_{1}+1\right)\left(2 l_{2}+1\right)\right]^{-1} \sum_{\substack{J_{i}^{\prime} K_{i}^{\prime} \\ J_{2}^{\prime} K_{2}^{\prime}}}\left(C_{J_{i}-K_{i} l_{1} l_{1}^{\prime}-K_{1}^{\prime}}^{J_{1}^{\prime}}\right)^{2}\left(C_{J_{2}-K_{2} l_{2} k_{2}}^{J_{2}^{\prime}-K_{2}^{\prime}}\right)^{2} f_{l_{1} l_{2} l}^{k_{1} k_{2}}
$$




$$
S_{2, f 2 i 2}=-\frac{2 r_{c}^{2}}{\hbar^{2} \overline{\mathrm{v}}^{2}} \sum_{\substack{l, l_{1} l \\ k_{2}}}(-1)^{\rho+l_{2}+l}\left[\left(2 l_{1}+1\right)\left(2 l_{2}+1\right)\right]^{-1} D\left(J_{i} J_{f} K_{i} K_{f} ; \rho l_{1}\right) \sum_{J_{2}^{\prime} K_{2}^{\prime}}\left(C_{J_{2} K_{2} L_{2} K_{2}}^{J^{\prime} K_{2}^{\prime}}\right)^{2} f_{l_{1} l_{2}}^{0 k_{2}},
$$

(in the second equation $k_{1}=0$ for $l_{1} \leq 2$ since this term is diagonal on the rotational quantum numbers of the active molecule). The so-called resonance functions $f_{1_{1} l_{l}^{l}}^{k_{1} k_{2}}$ have the same form as those given in Ref. [16] for $\mathrm{CH}_{3} \mathrm{X}-\mathrm{Y}_{2}$ but with the second (zero) superscript of the radial potential components replaced by $k_{2}$. The coefficients $D\left(J_{i} J_{f} K_{i} K_{f} ; \rho l_{1}\right)=$ $(-1)^{J_{i}+J_{f}} 2\left[\left(2 J_{i}+1\right)\left(2 J_{f}+1\right)\right]^{1 / 2}\left(C_{J_{i} K_{i} l_{1}}^{J_{K} K_{i}}\right)\left(C_{J_{f} K_{f} f_{1} 0}^{J_{f} K_{f^{\prime}}}\right) W\left(J_{i} J_{f} J_{i} J_{f} ; \rho l_{1}\right)$ depend in particular on the Racah coefficients $W\left(J_{i} J_{f} J_{i} J_{f} ; \rho l_{1}\right)$. To govern the classical trajectories, the isotropic potential is traditionally taken in the Lennard-Jones form with the parameters $\varepsilon=368.4 \mathrm{~K}$, $\sigma=3.584 \AA$ [38].

We have started our calculations with Eq. (4). Since the electrostatic dipole-dipole interactions yield clearly dominant contribution to the collisional broadening of $\mathrm{CH}_{3} \mathrm{Cl}$ $\mathrm{CH}_{3} \mathrm{Cl}$ lines [9] we have studied first the line widths resulting solely from this interaction, in order to establish the calculation parameters (maximal included values of the intermolecular distance, of the resonance parameter, etc.) required for the converged results. An example of calculated $K$-dependences is given in Fig. 6 for the transition $J=4 \rightarrow 5$. As can be seen from this figure, the intermolecular distances should be taken into account up to their very high values and the line widths are nearly converged with the maximal value of $35 \AA$. The convergence with respect to the resonance parameter is insured even for $k_{c \max }=20$ but in our calculations we kept $k_{c \max }=25$. Further we have tested the role of the other dipolequadrupole and quadrupole-quadrupole electrostatic contributions (the influence of these interactions for a maximal intermolecular distance of $15 \AA$ can be also seen in Fig. 6). These calculations show that adding dipole-quadrupole interactions $\left(V_{123}^{00 e}+V_{213}^{00 e}\right)$ increases the line broadening very slightly and that the quadrupole-quadrupole interaction $\left(V_{224}^{00 e}\right)$ is completely negligible. Therefore, for final calculations we have kept only three terms $V_{112}^{00 e}, V_{123}^{00 e}, V_{213}^{00 e}$ in the electrostatic part of the potential.

Figure 7 shows the $J$-dependence of line broadening calculated for $K=0$ and $K=7$ with these three electrostatic terms for various maximal values of the intermolecular distance. It can be stated from this figure that the electrostatic interactions at large intermolecular distances contribute significantly to the line widths at middle $J$-values (as well as at very low $J$-values) and even overestimate the line broadening for these $J$. This overestimation can be 
explained by the approximate character of classical trajectories governed solely by the isotropic part of the interaction potential and accumulation of errors when doing trajectory integrations up to very large distances required for the dipole-dipole interaction. Although the general behaviour of the experimentally observed $J$-dependences is correctly reproduced by the calculations with the electrostatic potential, the underestimated line broadening clearly visible in Fig. 7 for very high $J$ values (comparison with the measurements in the pure rotational band) seems to indicate (see Refs. $[17,39]$ ) that the long-range part of the intermolecular interactions is not sufficient and that the short-range forces should be included. We have completed therefore the leading $V_{112}^{00}$ term by the corresponding atom-atom interaction $V_{112}^{00 a-a}$ (other atom-atom terms were supposed to be negligible) and recalculated the line broadening coefficients. No observable change in the calculated values has been stated (Fig. 7), which means that the short-range interactions are of minor importance for the self-broadening of methyl chloride lines.

Since the converged line widths visibly overestimate the line broadening for middle $J$ values (Fig. 7), we have additionally sought for alternative models for the isotropic potential which governs the relative molecular trajectories and influences the second-order contributions $S_{2}$. Using the code available for the atom-atom radial potential components we have computed the isotropic potential issued from the atom-atom interactions. Figure 8 shows a comparison of this new (numerical) potential with the previous Lennard-Jones one. Despite the fact that in the region of small intermolecular distances $r \leq 5.5 \AA$ these potentials differ significantly, the line widths calculated with the numerical potential have practically the same values as that previously obtained with the Lennard-Jones model. It means that again only the long-range interactions are important for the $\mathrm{CH}_{3} \mathrm{Cl}-\mathrm{CH}_{3} \mathrm{Cl}$ system.

To resolve the problem of line-broadening overestimation (already mentioned in Ref. [9]) we have employed a solution proposed recently by Gomez et al. [40] which consists in applying a cut-off procedure on the maximal value of the intermolecular distance included in computations. As can be seen from Fig. 7, for the $K$-values studied in the present work a good agreement with the experimental data seems to be achieved for this cut-off realised at 22 Å.

The final point of our calculations is a comparison of line broadening coefficients computed with Eqs. (4) and (5). When Eq. (5) is used to calculate the line broadening with the cut-off at $22 \AA$, the computed values become too high and too distant from the experimental points, as it is shown in Fig. 9 for $K=0$. If the cut-off is realized at shorter intermolecular 
distances, the maximum of broadening clearly observed on the experimental points is more and more smoothed on the theoretical curves, and the calculated $J$-dependences become completely unrealistic. We have to conclude therefore that, despite a correct use of the cumulant expansion, the modified line widths expression of Eq. (5) leads to very strongly overestimated self-broadening coefficients which can not be corrected even by an artificial cut-off procedure. Consequently, it is Eq. (4) which is retained for final calculations (with the cut-off at $22 \AA$ ) given as supplementary data for $J$ and $K$ quantum numbers ranging from 0 to 60 and 0 to 25 , respectively.

\section{Comparison of results and discussion}

The recorded high-resolution spectra of the $v_{1}$ band have allowed the measurements of 527 self-broadening coefficients for $0 \leq J \leq 42$ and $0 \leq K \leq 11$ (See Table 2 and supplementary data). For the pure rotational band, 43 self-broadening coefficients have been retrieved (see Table 3). Comparisons between the measurements, the empirical modeling and the semiclassical calculations are presented for each set of transitions with same value of $K$ in Fig. 5 together with the measurements of Ref. [8]. The whole set of measurements is given in Fig. 10 with the empirical $J$-dependence present in HITRAN08 [25] coming from Ref. [6]. For each set of transitions with same $K$-value, a similar trend of the self-broadening coefficients is observed, with the maximum broadening occurring at $J=16$. However, contrary to the $\mathrm{CH}_{3} \mathrm{Br}$ [30] case, a strong increase of self-broadening coefficients is observed at low $J$-values (0-2) for $K=0$ or 1 .

With respect to the measurements issued from different experimental techniques, the agreement between results obtained for transitions of the $v_{1}$ band and for pure rotational transitions is very good. The various measurements are consistent except maybe for the $J=6$ measurements for which a systematic discrepancy is observed between the (FT) and (FM) results. The measurements of Ref. [7] for 29 transitions of the $v_{3}$ band have been plotted in Fig. 5, showing a very good consistency between the various measurements. No significant vibrational dependence has been observed allowing thus to extrapolate our measurements and calculations to other vibrational bands of $\mathrm{CH}_{3} \mathrm{Cl}$. For the $v_{1}$ band, measurements have been obtained for both $\mathrm{CH}_{3}{ }^{35} \mathrm{Cl}$ and $\mathrm{CH}_{3}{ }^{37} \mathrm{Cl}$ isotopologues showing no significant discrepancy between the two isotopologues.

As seen in Fig. 5, both the empirical model described in Section 4 and the semiclassical calculation developed in Section 5 are in good agreement with the measurements. 
However, it can be notice a small systematic discrepancy for high $J$-values ( $>35$ ) between the empirical model and the semi-classical calculation. Deviations are also observed when $K$ is close to $J$.

The high dispersion of the measurements presented in Fig. 10 is not due to the experimental uncertainties but to the significant $K$-dependence of the self-broadening coefficients which is especially pronounced for $K$ approaching $J$. As it can be observed in Fig. 5 , the comparison between results obtained by different experimental methods shows a good agreement, with maximal average deviation not exceeding $10 \%$.

The present results showing and modelling $J$ - and $K$-rotational dependences of the self-broadening coefficients of $\mathrm{CH}_{3} \mathrm{Cl}$ make obvious that the polynomial expression of Chackerian et al. [6] that did not took into account the $K$-rotational dependence could be replaced in HITRAN08 database by the results of the present work.

\section{Conclusion}

Extensive measurements of $\mathrm{CH}_{3} \mathrm{Cl}$ self-broadening coefficients of transitions belonging to the $v_{1}$ band (2930-3001 $\left.\mathrm{cm}^{-1}\right)$ and to the rotational band $\left(5-45 \mathrm{~cm}^{-1}\right)$ have been performed in order to study both the rotational and vibrational dependences. Two different experimental techniques, using $\mathrm{CW}-\mathrm{THz}$ photomixing and frequency-multiplication spectrometers, allowed to achieve measurements for pure rotational transitions, whereas the infrared region was studied with a FT spectrometer. No vibrational nor isotopologue dependences has been observed, but similar $J$ - and $K$ - rotational dependences were observed for pure rotational transitions and transitions of the $v_{1}$ band.

For practical use, an empirical polynomial model has been proposed whereas a semiclassical approach, involving exact classical trajectories, an interaction potential with shortrange forces, and a rigorous treatment of the active molecule as a symmetric top, has been used to calculate self-broadening coefficients for $J$ and $K$ quantum numbers ranging from 0 to 60 and 0 to 25, respectively. Because of very strong dipole-dipole interactions in the $\mathrm{CH}_{3} \mathrm{Cl}$ $\mathrm{CH}_{3} \mathrm{Cl}$ system, the semi-classical results have had to be corrected via an artificial cut-off procedure in order to match the experimental data near the maximum of broadening. It will be very interesting to extend this study to other halogenated $\mathrm{CH}_{3} \mathrm{X}$ compounds with $(\mathrm{X}=\mathrm{F}, \mathrm{Br}, \mathrm{I})$. By a systematic comparison of the self-broadening coefficients, we should particularly be able 
to understand the influence of the halogen nucleus and the molecular field gradient on the rotational $J$ and $K$ dependences.

\section{Acknowledgments}

FR acknowledges Prof. Laurent Margulès and Dr. Roman A. Motyenko for experimental assistance. The financial support of MG is provided by the European Commission via the Interreg IVA-2 seas (CleanTech project). The LPCA and PhLAM laboratories are supported by the Région Nord-Pas de Calais and the Fond Européen de Développement Economique des Régions (FEDER). LPCA participates in the Institut de Recherche en ENvironnement Industriel (IRENI) which is financed by the Communauté Urbaine de Dunkerque. 


\section{References}

[1] Fraser SA, Frase PJ, Butler JH, Connell PS, Cunnold DM, Daniel JS, Derwent RG, Lal S, McCulloch A, Oram DE, Reeves CE, Sanhueza E, Steele LP, Velders GJM, Weiss RF, Zander RJ. Controlled substances and other source gases. Chapter 1 in Scientific Assessment of Ozone Depletion: 2002, Global Ozone Research and Monitoring Project-Report No. 47, World Meteorological Organization, Geneva (2003).

[2] Yoshida Y. Global sources and distribution of atmospheric methyl chloride. thesis (2006).

[3] Khalil MAK, Moore RM, Harper DB, Lobert JM, Erickson DJ, Koropalov V, Sturges WT, Keene WC. Natural emissions of chlorine-containing gases: Reactive Chlorine Emissions Inventory. J. Geophys. Res. 1999;104:8333-46.

[4] Keene WC, Khalil MAK, Erickson III DJ, McCulloch A, Graedel TE, Lobert JM, Aucott ML, Gong SL, Harper DH, Kleiman G, Midgley P, Moore RM, Seuzaret C, Sturges WT, Benkovitz CM, Koropalov V, Barrie LA, Li YF. Composite global emissions of reactive chlorine from anthropogenic and natural sources: Reactive Chlorine Emissions Inventory. J. Geophys. Res. 1999;104:8429-40.

[5] Kaley AW, Weigum N, McElcheran C, Taylor JR. Global methyl chloride measurements from the ACE-FTS instrument. International Symposium on Molecular Spectroscopy Department of Chemistry The Ohio State University, TI-09; 2009.

[6] Chackerian C, Jr, Brown LR, Lacome N, Tarrago G. Methyl Chloride $v_{5}$ Region Lineshape Parameters and Rotational Constants for the $v_{2}, v_{5}$, and $2 v_{3}$ Vibrational Bands. J. Mol. Spectrosc. 1998;191:148-57.

[7] Bouanich JP, Blanquet G, Walrand J. Diode-laser measurements of self-broadening coefficients and line strength in the $v_{3}$ band of $\mathrm{CH}_{3}{ }^{35} \mathrm{Cl}$. J. Quant. Spectrosc. Radiat. Transfer 1994;51:573-8.

[8] Blanquet G, Coupe P, Walrand J, Bouanich JP. Determination of broadening coefficients and intensities for overlapping spectral lines with application to the ${ }^{Q} R(3, K)$ lines in the $v_{3}$ band of $\mathrm{CH}_{3}{ }^{35} \mathrm{Cl}$. J. Quant. Spectrosc. Radiat. Transfer 1994;51:671-8.

[9] Blanquet G, Walrand J, Populaire JP, Bouanich JP. Self-broadening coefficients and line strength in the $v_{3}$ band of $\mathrm{CH}_{3}{ }^{35} \mathrm{Cl}$ at low temperature. J. Quant. Spectrosc. Radiat. Transfer 1995;53:211-9.

[10] Roberts JA, Parsons RW. Self and foreign gas broadening of $J=0-1$ line in rotational spectrum of methyl chloride. J. Mol. Spectrosc. 1966:20: 195-7.

[11] Chikashi Y, Toshiaki S, Eizi H. Pressure Broadening Spectroscopy of Formaldehyde, Ammonia, lsocyanic Acid, Trimethylene Sulfide, and Methyl Chloride Using Far-Infrared Lasers J. Mol. Spectrosc. 1975:54: 261-74.

[12] Harde H, Chevile RA, Grischkowsky D. Terahertz studies of collision-broadened rotational lines. J. Phys. Chem. A, 1997:101:3646-60.

[13] Bray C, Perrin A, Jacquemart D, Lacome N. The $v_{1}, v_{4}$ and $3 v_{6}$ bands of methyl chloride in the $3.4 \mu \mathrm{m}$ region: line positions and intensities. J. Quant. Spectrosc. Radiat. Transfer 2011;112:24462462.

[14] Mouret G, Hindle F, Cuisset A, Yang C, Bocquet R, Lours M, Rovera D. THz photomixing synthesizer based on a fiber frequency comb. Opt Expr 2009;17:22031-40. 
[15] Hindle F, Mouret G, Eliet S, Guinet M, Cuisset A, Bocquet R, Yasui T, Rovera D. Widely tunable THz synthesizer. Appl. Phys. B 2011;104:763-8.

[16] Buldyreva J, Guinet M, Eliet S, Hindle F, Mouret G, Bocquet R, Cuisset A. Theoretical and experimental studies of $\mathrm{CH}_{3} \mathrm{X}-\mathrm{Y}_{2}$ rotational line shapes for atmospheric spectra modelling: application to room-temperature $\mathrm{CH}_{3} \mathrm{Cl}-\mathrm{O}_{2}$. Phys. Chem. Chem. Phys. 2011;13:20326-34

[17] Guinet M, Rohart F, Buldyreva J, Gupta V, Eliet S, Motiyenko R, Margulès L, Cuisset A, Hindle F, Mouret G. Experimental studies by complementary terahertz techniques and semi-classical calculations of $\mathrm{N}_{2}$-broadening coefficients of $\mathrm{CH}_{3} \mathrm{Cl}$. J. Quant. Spectrosc. Radiat. Transfer 2012;113:1113-26.

[18] Buldyreva J, Rohart F. Experimental and theoretical studies of room-temperature sub-millimeter $\mathrm{CH}_{3} \mathrm{Cl}$ line shapes broadened by $\mathrm{H}_{2}$. Mol. Phys. DOI:10.1080/00268976.2012.684895, in press (2012).

[19] Pickett HM, Poynter RL, Cohen EA, Delitsky ML, Pearson JC, Müller HSP. Submillimeter, millimeter, and microwave spectral line catalog. J. Quant. Spectrosc. Radiat. Transfer 1998;60:883-90.

[20] Buffa G, Di Lieto A, Minguzzi P, Tarrini O, Tonelli M. Nuclear-quadrupole effects in the pressure broadening of molecular lines. Phys. Rev. A 1988;37:3790-4.

[21] Belli S, Buffa G, Tarrini O. Collisional coupling between hyperfine and Stark components of molecular spectra. Phys. Rev. A 1997;55:83-90.

[22] Jacquemart D, Mandin JY, Dana V, Picqué N, Guelachvili G. A multispectrum fitting procedure to deduce molecular line parameters. Application to the $3-0$ band of ${ }^{12} \mathrm{C}^{16} \mathrm{O}$. Eur. Phys. J. D 2001;14:55-69.

[23] Guinet M, Jeseck P, Mondelain D, Pepin I, Janssen C, Camy-Peyret C, Mandin JY. Absolute measurements of intensities, positions and self-broadening coefficients of $\mathrm{R}$ branch transitions in the $v_{2}$ band of ammonia. J. Quant. Spectrosc. Radiat. Transfer 2011;112:1950-60.

[24] Colmont JP, Rohart F, Wlodarczak G, Bouanich JP. K-dependence and temperature dependence of $\mathrm{N}_{2}$ - and $\mathrm{O}_{2}$-broadening coefficients for the $\mathrm{J}=14-13$ transition of methyl chloride $\mathrm{CH}_{3}{ }^{35} \mathrm{Cl}$. J. Mol. Struct. 2006;780-781:268-76.

[25] Rothman LS, Gordon IE, Barbe A, Benner DC, Bernath PF, Birk M, Boudon V, Brown LR, Campargue A, Champion JP, Chance, K, Coudert L, Dana V, Devi VM, Fally S, Flaud JM, Gamache RR, Goldman A, Jacquemart D, Kleiner I, Lacome N, Lafferty WJ, Mandin JY, Massie ST, Mikhailenko SN, Miller CE, Moazzen-Ahmadi N, Naumenko OV, Nikitin AV, Orphal J, Perevalov VI, Perrin A, Predoi-Cross A, Rinsland CP, Rotger M, Simeckova M, Smith MAH, Sung K, Tashkun SA, Tennyson J, Toth RA, Vandaele AC, Vander Auwera J. The HITRAN 2008 molecular spectroscopic database. J. Quant. Spectrosc. Radiat. Transfer 2009;110:533-72.

[26] Dore L. Using Fast Fourier Transform to compute the line shape of frequency-modulated spectral profiles. J. Mol. Spectrosc. 2003;221:93-8.

[27] Hartmann JM, Boulet C, Robert D. Collisional Effects on Molecular Spectra. Laboratory Experiments and Models, Consequences for Applications (Elsevier, Amsterdam, 2008).

[28] De Vizia MD, Rohart F, Castrillo A, Fasci E, Moretti L, Gianfrani L. Speed-dependent effects in the near-infrared spectrum of self-colliding $\mathrm{H}_{2}{ }^{18} \mathrm{O}$ molecules. Phys. Rev. A 2011;83:052506. 
[29] Looney JP. Comprehensive theory for broadening, shifting and narrowing of $\mathrm{HF}$ and $\mathrm{HCl}$ fundamental band absorption profiles. Ph.D. Thesis, Pennsylvania State University, Philadelphia, 1987.

[30] Jacquemart D, Kwabia Tchana F, Lacome N, Kleiner I. A complete set of line parameters for $\mathrm{CH}_{3} \mathrm{Br}$ in the 10- $\mu \mathrm{m}$ spectral region. J. Quant. Spectrosc. Radiat. Transfer 2007;105:264-302.

[31] Bray C, Jacquemart D, Buldyreva J, Lacome N, Perrin A. The $\mathrm{N}_{2}$-broadening coefficients of methyl chloride at room temperature. J. Quant. Spectrosc. Radiat. Transfer 2012;113:1102-1112.

[32] Robert D, Bonamy J. Short range force effects in semiclassical molecular line broadening calculations. J. Phys. 1979;40:923-43.

[33] Ma Q, Tipping RH, Boulet C. Modification of the Robert-Bonamy formalism in calculating Lorentzian half-widths and shifts. J. Quant. Spectrosc. Radiat. Transfer 2007;103:588-96.

[34] Antony BK, Gamache PR, Szembek CD, Niles DL, Gamache RR. Modified complex RobertBonamy formalism calculations for strong to weak interacting systems. Mol. Phys. $2006 ; 104$ :2791-9.

[35] Landau LD, Lifshits EM. Course of Theoretical Physics v.1, Pergamon, Oxford 1976.

[36] Buldyreva J, Nguyen L. Extension of the exact trajectory model to the case of asymmetric tops and its application to infrared nitrogen-broadened linewidths of ethylene. Phys. Rev. A 2008:77;042720.

[37] Gray CG, K.E. Gubbins, Theory of Molecular Fluids, Oxford University Press, London/New York, 1984.

[38] Bouanich JP, Blanquet G, Walrand J. Theoretical $\mathrm{O}_{2}$ - and $\mathrm{N}_{2}$-Broadening coefficients of $\mathrm{CH}_{3} \mathrm{Cl}$ spectral lines. J. Mol. Spectrosc. 1993:161;416-26.

[39] Motiyenko RA, Margulès L, Alekseev EA, Guillemin JC, Demaison J. Centrifugal distortion analysis of the rotational spectrum of aziridine: Comparison of different Hamiltonians. J. Mol. Spectrosc. 2010;264:94-9.

[40] Gomez L, Jacquemart D, Bouanich JP, Boussetta Z, Aroui H. Theoretical calculations of selfbroadening coefficients in the $v_{6}$ band of $\mathrm{CH}_{3} \mathrm{Br}$. J. Quant. Spectrosc. Radiat. Transfer 2010:111;125261. 


\section{Table 1:}

Experimental conditions and characteristics of the recorded spectra.

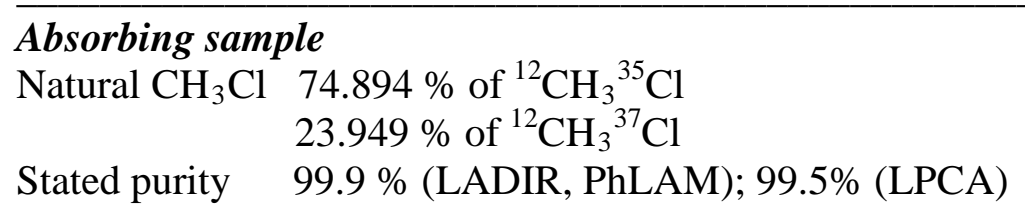

\section{LADIR (FT spectroscopy)}

Unapodized apparatus function

Nominal aperture radius

$0.75 \mathrm{~mm}$

Effective aperture radius

$0.80 \mathrm{~mm}$

Collimator focal length

$418 \mathrm{~mm}$

Experimental conditions

\begin{tabular}{llll}
\hline$\#$ & $\begin{array}{c}\mathrm{CH}_{3} \mathrm{Cl} \text { pressure } \\
(\mathrm{mbar})\end{array}$ & $\begin{array}{c}\text { Resolution }^{\mathrm{a}} \\
\left(\mathrm{cm}^{-1}\right)\end{array}$ & $\begin{array}{c}\text { Absorption path } \\
(\mathrm{cm})\end{array}$ \\
\hline 1 & $0.542_{0}$ & 0.008 & $30.00 \pm 0.05$ \\
2 & $0.621_{0}$ & 0.008 & $30.00 \pm 0.05$ \\
$3^{\mathrm{b}}$ & $0.702_{0}$ & 0.008 & $30.00 \pm 0.05$ \\
4 & 1.51 & 0.008 & $30.00 \pm 0.05$ \\
5 & 3.30 & 0.01 & $30.00 \pm 0.05$ \\
6 & 7.10 & 0.01 & $30.00 \pm 0.05$
\end{tabular}

\footnotetext{
${ }^{\mathrm{a}}$ Resolution as defined by Bruker = 0.9/MOPD (Maximum Optical Path Difference).

${ }^{\mathrm{b}}$ For this spectra no optical filter has been used (see text).
}

\section{LPCA (PM spectroscopy)}

Modulation amplitude: $350 \mathrm{~Hz}$ (mechanical chopper)

Detection time constant: $300 \mathrm{~ms}$

Frequency steps: $800 \mathrm{kHz}$ at a $1 \mathrm{~Hz}$ rate

$\mathrm{CH}_{3} \mathrm{Cl}$ pressures: 0.1 Torr $<P<1$ Torr

Absorption path: $25.0 \pm 0.2 \mathrm{~cm}(J=31)$

$$
\begin{aligned}
& 65.0 \pm 0.2 \mathrm{~cm}(J=37 \text { and } J=40) \\
& 85.0 \pm 0.2 \mathrm{~cm}(J=45) \\
& 120.0 \pm 0.2 \mathrm{~cm}(J=50)
\end{aligned}
$$

\section{PhLAM (FM spectroscopy)}

Modulation frequency: $10.5 \mathrm{kHz}$

Modulation amplitude: $180 \mathrm{kHz}$

Detection at twice modulation frequency: $21 \mathrm{kHz}$

Detection time constant: $10 \mathrm{~ms}$

Frequency steps: $15.6 \mathrm{kHz}$ at a $30 \mathrm{~Hz}$ rate

$\mathrm{CH}_{3} \mathrm{Cl}$ pressures: 19.9 , 39.3, 61.7, 81.6 and 104.6 mTorr

Absorption path: $110.0 \pm 0.5 \mathrm{~cm}$ 
Table 2:

Sample of self-broadening coefficients measured for transitions of the $v_{1}$ band.

\begin{tabular}{|c|c|c|c|c|c|c|c|c|c|c|c|c|c|}
\hline \multirow{3}{*}{$\frac{\text { ISO }}{241}$} & \multicolumn{6}{|c|}{ Assignment } & \multirow[t]{2}{*}{ Position } & \multirow[t]{2}{*}{ Obs } & \multirow[t]{2}{*}{ Fit error } & \multirow[t]{2}{*}{ Calc1 } & \multirow[t]{2}{*}{$\%$} & \multirow[t]{2}{*}{ Calc2 } & \multirow[t]{2}{*}{$\%$} \\
\hline & \multicolumn{3}{|c|}{ Upper state } & \multicolumn{3}{|c|}{ Lower state } & & & & & & & \\
\hline & 6 & 2 & $E$ & 7 & 2 & E & 2961.34247 & 0.4188 & $\odot .0032$ & 0.423 & -1.0 & 0.443 & -5.5 \\
\hline 242 & 6 & 2 & $E$ & 7 & 2 & $E$ & 2961.41703 & 0.4391 & 0.0029 & 0.423 & 3.7 & 0.443 & -0.9 \\
\hline 241 & 6 & 1 & $E$ & 7 & 1 & $E$ & 2961.50876 & 0.3560 & ๑. . 0196 & $\odot .432$ & -21.3 & 0.448 & -20.5 \\
\hline 242 & 6 & 1 & $E$ & 7 & 1 & $E$ & 2961.58341 & 0.4680 & $\odot .0040$ & 0.432 & 7.7 & 0.448 & 4.5 \\
\hline 242 & 6 & $\odot$ & A1 & 7 & $\odot$ & $\mathrm{A} 2$ & 2961.63878 & 0.4265 & 0.0049 & 0.435 & -2.0 & 0.449 & -5.0 \\
\hline 241 & 5 & 3 & A & 6 & 3 & A & 2961.95114 & $\odot .4164$ & $\odot .0016$ & 0.385 & 7.5 & 0.418 & -0.4 \\
\hline 242 & 5 & 3 & A & 6 & 3 & A & 2962.01196 & 0.3720 & 0.0027 & 0.385 & -3.5 & 0.418 & -11.0 \\
\hline 242 & 5 & 1 & $E$ & 6 & 1 & $E$ & 2962.45511 & 0.4252 & 0.0032 & 0.418 & 1.7 & 0.435 & -2.3 \\
\hline 241 & 4 & 2 & $\mathrm{E}$ & 5 & 2 & $\mathrm{E}$ & 2963.11400 & 0.4036 & $\odot .0 \odot 27$ & 0.392 & 2.9 & 0.423 & -4.6 \\
\hline 242 & 4 & 2 & $E$ & 5 & 2 & E & 2963.16123 & 0.3668 & 0.0066 & $\odot .392$ & -6.9 & 0.423 & -13.3 \\
\hline 241 & 3 & 3 & $A$ & 4 & 3 & $A$ & 2963.72286 & 0.3524 & 0.0031 & 0.347 & 1.5 & 0.394 & -10.6 \\
\hline 242 & 3 & 3 & $A$ & 4 & 3 & $A$ & 2963.75643 & 0.3338 & $\odot .0 \odot 67$ & 0.347 & -4.0 & $\odot .394$ & -15.3 \\
\hline 241 & 3 & 2 & $E$ & 4 & 2 & $E$ & $2964.000 \odot 2$ & 0.3864 & 0.0032 & 0.384 & $\odot .6$ & 0.423 & -8.7 \\
\hline 242 & 3 & 2 & $E$ & 4 & 2 & $E$ & 2964.03370 & 0.3223 & ๑. .0081 & 0.384 & -19.1 & 0.423 & -23.8 \\
\hline 241 & 3 & 1 & $\mathrm{E}$ & 4 & 1 & $\mathrm{E}$ & 2964.16641 & 0.4039 & $\odot .0 \odot 37$ & 0.407 & -0.8 & 0.437 & -7.6 \\
\hline 241 & 2 & 2 & $E$ & 3 & 2 & $E$ & 2964.88629 & 0.3890 & 0.0068 & 0.385 & 1.0 & 0.420 & -7.4 \\
\hline 242 & 2 & 2 & $E$ & 3 & 2 & $E$ & 2964.90620 & 0.3636 & 0.0081 & 0.385 & -5.9 & 0.420 & -13.4 \\
\hline 241 & 1 & 1 & $\mathrm{E}$ & 2 & 1 & E & 2965.93925 & 0.4278 & $\odot .0235$ & 0.440 & -2.9 & 0.447 & -4.3 \\
\hline 242 & 1 & 1 & $E$ & 2 & 1 & $E$ & 2965.94577 & 0.4702 & 0.0112 & 0.440 & 6.4 & 0.447 & 5.2 \\
\hline 241 & 1 & $\odot$ & $\mathrm{A} 2$ & 2 & $\odot$ & A1 & 2965.99463 & 0.4764 & 0.0190 & 0.454 & 4.7 & 0.461 & 3.3 \\
\hline
\end{tabular}

In Column 1, ISO refers to the $\mathrm{CH}_{3}{ }^{35} \mathrm{Cl}$ (241) and $\mathrm{CH}_{3}{ }^{37} \mathrm{Cl}$ (242) isotopic species (AFGL notation used in HITRAN). Columns 2 to 7 give the assignment ( $J, K$ and symmetry type) of the upper and lower state of transitions. Note that " $A$ " stands for overlapping $A_{1} \leftarrow A_{2}$ and $A_{2} \leftarrow A_{1}$ transitions. Column 8 corresponds to the experimental line positions (in $\mathrm{cm}^{-1}$ ). Columns $9-10$ are the measured self-broadening coefficients and their corresponding fitting uncertainties in $\mathrm{cm}^{-1} \cdot \mathrm{atm}^{-1}$ at 296K. Column 11 and 13 are the calculated broadening coefficients (in $\mathrm{cm}^{-1}$.atm ${ }^{-1}$ at $296 \mathrm{~K}$ ) obtained respectively with the empirical model (section 4) and the semi-classical model (section 5). The columns 12 and 14 represent the differences between the observed and calculated widths in percent (Obs-Calc/Obs). The complete Table is available as supplementary material. 
Table 3:

Measured self-broadening coefficients (in $\mathrm{cm}^{-1} \cdot \mathrm{atm}^{-1}$ at 296K) for pure rotational transitions. Between parentheses, are given the uncertainties in units of the last digit. For $J=6$ values the observed widths have been obtained using the frequency modulation experiment. For the other values of $J$, the photomixing spectrometer has been employed.

\begin{tabular}{|c|c|c|c|c|c|c|}
\hline$J$ & $K$ & Obs & Calc1 & $\%$ & Calc2 & $\%$ \\
\hline 6 & 0 & $0.452(10)$ & 0.422 & 6.6 & 0.437 & 3.3 \\
\hline 6 & 1 & $0.477(12)$ & 0.418 & 12.4 & 0.435 & 8.8 \\
\hline 6 & 2 & $0.438(4)$ & 0.406 & 7.3 & 0.429 & 2.1 \\
\hline 6 & 3 & $0.444(5)$ & 0.385 & 13.3 & 0.418 & 5.9 \\
\hline 6 & 4 & $0.376(7)$ & 0.356 & 5.3 & 0.400 & -6.4 \\
\hline 6 & 5 & $0.332(5)$ & 0.320 & 3.6 & 0.371 & -11.7 \\
\hline \multirow[t]{2}{*}{6} & 6 & $0.313(3)$ & 0.274 & 12.5 & 0.317 & -1.3 \\
\hline & & & & -- & & -- \\
\hline 31 & 0 & $0.368(15)$ & 0.370 & -0.5 & 0.349 & 5.2 \\
\hline 31 & 1 & $0.358(14)$ & 0.369 & -3.1 & 0.349 & 2.5 \\
\hline 31 & 2 & $0.363(15)$ & 0.368 & -1.4 & 0.349 & 3.9 \\
\hline 31 & 3 & $0.363(15)$ & 0.366 & -0.8 & 0.349 & 3.9 \\
\hline 31 & 4 & $0.353(14)$ & 0.363 & -2.8 & 0.348 & 1.4 \\
\hline 31 & 6 & $0.358(14)$ & 0.354 & 1.1 & 0.347 & 3.1 \\
\hline 31 & 7 & $0.341(31)$ & 0.349 & -2.3 & 0.346 & -1.5 \\
\hline 31 & 9 & $0.341(31)$ & 0.335 & 1.8 & 0.344 & -0.9 \\
\hline \multirow[t]{2}{*}{31} & 12 & $0.337(30)$ & -- & -- & 0.339 & -0.6 \\
\hline & & & & -- & & -- \\
\hline 37 & 0 & $0.290(12)$ & 0.281 & 3.1 & 0.247 & 14.8 \\
\hline 37 & 1 & $0.284(11)$ & 0.281 & 1.1 & 0.247 & 13.0 \\
\hline 37 & 2 & $0.287(12)$ & 0.280 & 2.4 & 0.247 & 13.9 \\
\hline 37 & 3 & $0.280(11)$ & 0.279 & 0.4 & 0.247 & 11.8 \\
\hline 37 & 4 & $0.275(25)$ & 0.276 & -0.4 & 0.246 & 10.5 \\
\hline 37 & 6 & $0.258(10)$ & 0.270 & -4.7 & 0.246 & 4.7 \\
\hline 37 & 9 & $0.254(23)$ & 0.256 & -0.8 & 0.245 & 3.5 \\
\hline \multirow[t]{2}{*}{37} & 12 & $0.246(22)$ & --- & -- & 0.242 & 1.6 \\
\hline & & & & -- & & -- \\
\hline 40 & 2 & $0.252(10)$ & 0.247 & 2.0 & 0.207 & 17.9 \\
\hline 40 & 3 & $0.258(10)$ & 0.246 & 4.7 & 0.207 & 19.8 \\
\hline 40 & 4 & $0.241(10)$ & 0.244 & -1.2 & 0.207 & 14.1 \\
\hline 40 & 6 & $0.231(9)$ & 0.239 & -3.5 & 0.206 & 10.8 \\
\hline 40 & 9 & $0.214(19)$ & 0.227 & -6.1 & 0.205 & 4.2 \\
\hline \multirow[t]{2}{*}{40} & 12 & $0.206(19)$ & --- & -- & 0.204 & 1.0 \\
\hline & & & & -- & & -- \\
\hline 45 & 0 & $0.207(8)$ & 0.202 & 2.4 & 0.158 & 23.7 \\
\hline 45 & 1 & $0.207(8)$ & 0.201 & 2.9 & 0.158 & 23.7 \\
\hline 45 & 2 & $0.201(8)$ & 0.201 & 0 & 0.158 & 21.4 \\
\hline 45 & 4 & $0.189(8)$ & 0.198 & -4.8 & 0.158 & 16.4 \\
\hline 45 & 6 & $0.198(8)$ & 0.194 & 2.0 & 0.157 & 20.7 \\
\hline 45 & 9 & $0.174(16)$ & 0.185 & -6.3 & 0.157 & 9.8 \\
\hline 45 & 12 & $0.176(16)$ & --- & -- & 0.156 & 11.4 \\
\hline
\end{tabular}


Table 3 (continued)

Measured self-broadening coefficients (in $\mathrm{cm}^{-1} \cdot \mathrm{atm}^{-1}$ at 296K) for pure rotational transitions. Between parentheses, are given the uncertainties in units of the last digit. For $J=6$ values the observed widths have been obtained using the frequency-modulation experiment. For the other values of $J$, the photo-mixing spectrometer has been employed.

$\begin{array}{llllrll}50 & 0 & 0.158(6) & 0.160 & -1.3 & 0.127 & 19.6 \\ 50 & 1 & 0.160(6) & 0.160 & 0 & 0.127 & 20.6 \\ 50 & 2 & 0.159(6) & 0.159 & 0 & 0.127 & 20.1 \\ 50 & 6 & 0.156(6) & 0.154 & 1.3 & 0.127 & 18.6 \\ 50 & 9 & 0.145(13) & 0.147 & -1.4 & 0.126 & 13.1 \\ 50 & 12 & 0.141(13) & --- & -- & 0.126 & 10.6\end{array}$

Calc1 and Calc2 are the calculated broadening coefficients (in $\mathrm{cm}^{-1} \cdot \mathrm{atm}^{-1}$ at 296K) obtained respectively with the empirical model (section 4) and the semi-classical model (section 5). The \% columns refer to the differences in \% between the observed and calculated widths (Obs-Calc/Obs) $\times 100$. 
Table 4:

$a_{J}^{0}$ and $a_{J}^{2}$ parameters (in $\mathrm{cm}^{-1} \cdot \mathrm{atm}^{-1}$ at 296K) obtained from Eq. (3). Between parentheses, are given the standard deviation (1SD) in units of the last digit.

\begin{tabular}{|c|c|c|c|c|}
\hline \multirow[b]{2}{*}{$J$} & \multicolumn{2}{|c|}{$a_{J}^{0}$} & \multicolumn{2}{|c|}{$a_{J}^{2}\left(\right.$ in $\left.10^{-3}\right)$} \\
\hline & $\begin{array}{l}\text { Fitted } \\
\text { values }\end{array}$ & $\begin{array}{l}\text { Smoothed } \\
\text { values }\end{array}$ & $\begin{array}{l}\text { Fitted } \\
\text { values }\end{array}$ & $\begin{array}{l}\text { Smoothed } \\
\text { values }\end{array}$ \\
\hline 0 & -- & 0.5903 & -- & \\
\hline 1 & -- & 0.5078 & -- & -19 \\
\hline 2 & $0.472(17)$ & 0.4544 & $-20.3(84)$ & -14 \\
\hline 3 & $0.3978(92)$ & 0.4249 & $-2.8(18)$ & -10 \\
\hline 4 & $0.400(12)$ & 0.4144 & $-7.0(17)$ & -7.5 \\
\hline 5 & $0.424(26)$ & 0.4144 & $-5.22(68)$ & -5.5 \\
\hline 6 & $0.4232(40)$ & 0.4221 & $-5.2(16)$ & -4.1 \\
\hline $6 *$ & $0.4504(48)$ & " & $-3.32(47)$ & $"$ \\
\hline 7 & $0.4316(80)$ & 0.4351 & $-2.04(53)$ & -3.0 \\
\hline 8 & $0.455(15)$ & 0.4515 & $-2.50(89)$ & -2.2 \\
\hline 9 & $0.486(17)$ & 0.4695 & $-3.10(82)$ & -1.7 \\
\hline 10 & $0.483(12)$ & 0.4878 & $-1.80(60)$ & -1.4 \\
\hline 11 & $0.505(13)$ & 0.5053 & $-0.66(73)$ & -1.2 \\
\hline 12 & $0.512(11)$ & 0.5211 & $-0.96(24)$ & -1.1 \\
\hline 13 & $0.558(18)$ & 0.5347 & $-1.19(24)$ & -1.0 \\
\hline 14 & $0.533(19)$ & 0.5454 & $-0.58(32)$ & -0.96 \\
\hline 15 & $0.551(15)$ & 0.5532 & $-0.67(25)$ & -0.93 \\
\hline 16 & $0.568(21)$ & 0.5579 & $-1.10(25)$ & -0.90 \\
\hline 17 & $0.555(38)$ & 0.5593 & $-0.79(50)$ & -0.86 \\
\hline 18 & $0.547(14)$ & 0.5577 & $-0.96(23)$ & -0.82 \\
\hline 19 & $0.556(18)$ & 0.5531 & $-0.88(23)$ & -0.78 \\
\hline 20 & $0.5352(26)$ & 0.5465 & $0.005(90)$ & -0.74 \\
\hline 21 & $0.522(22)$ & 0.5366 & $-0.35(41)$ & -0.70 \\
\hline 22 & $0.532(17)$ & 0.5244 & $-0.36(69)$ & -0.66 \\
\hline 23 & $0.504(18)$ & 0.5103 & $-0.34(32)$ & -0.64 \\
\hline 24 & $0.505(15)$ & 0.4947 & $-0.87(24)$ & -0.61 \\
\hline 25 & - & 0.4779 & -- & -0.58 \\
\hline 26 & $0.483(10)$ & 0.4602 & $-1.20(18)$ & -0.54 \\
\hline 27 & $0.449(11)$ & 0.4420 & $-0.73(22)$ & -0.51 \\
\hline 28 & $0.420(13)$ & 0.4237 & $-0.12(31)$ & -0.49 \\
\hline 29 & $0.4051(15)$ & 0.4053 & $0.12(57)$ & -0.47 \\
\hline 30 & $0.393(13)$ & 0.3873 & $-0.36(33)$ & -0.45 \\
\hline 31 & $0.360(14)$ & 0.3698 & $0.08(38)$ & -0.425 \\
\hline $31^{* *}$ & $0.3612(26)$ & " & $-0.21(19)$ & " \\
\hline 32 & $0.357(11)$ & 0.3529 & $0.08(35)$ & -0.40 \\
\hline 33 & $0.3267(88)$ & 0.3369 & $-0.19(27)$ & -0.38 \\
\hline 34 & $0.3094(16)$ & 0.3217 & $0.24(44)$ & -0.36 \\
\hline 35 & $0.3180(14)$ & 0.3074 & $-0.249(77)$ & -0.34 \\
\hline 36 & $0.2945(10)$ & 0.2940 & $-0.090(64)$ & -0.32 \\
\hline 37 & $0.2834(16)$ & 0.2815 & $-0.095(95)$ & -0.31 \\
\hline $37 * *$ & $0.2865(59)$ & " & $-0.645(23)$ & " \\
\hline 38 & $0.2658(20)$ & 0.2698 & $-0.604(61)$ & -0.29 \\
\hline 39 & -- & 0.2588 & -- & -0.28 \\
\hline $40 * *$ & $0.2512(59)$ & 0.2485 & $-0.38(13)$ & -0.265 \\
\hline 41 & -- & 0.2386 & -- & -0.255 \\
\hline 42 & -- & 0.2290 & -- & -0.24 \\
\hline 43 & -- & 0.2197 & -- & -0.23 \\
\hline 44 & -- & 0.2106 & -- & -0.215 \\
\hline $45^{* *}$ & $0.2017(40)$ & 0.2016 & $-0.23(10)$ & -0.205 \\
\hline 46 & -- & 0.1926 & -- & -0.195 \\
\hline 47 & -- & 0.1838 & -- & -0.185 \\
\hline 48 & -- & 0.1752 & -- & -0.175 \\
\hline 49 & -- & 0.1671 & -- & -0.165 \\
\hline $50 * *$ & $0.1595(35)$ & 0.1599 & $-0.131(82)$ & -0.155 \\
\hline
\end{tabular}

* Results from frequency-modulation spectrometer measurements.

** Results from photo-mixing spectrometer measurements. 


\section{Figure 1:}

$K=0,1$ and 2 components of the $J=40$ multiplet of $\mathrm{CH}_{3}{ }^{35} \mathrm{Cl}$ measured from four $\mathrm{CW}-\mathrm{THz}$ spectra recorded at $P=0.228,0.1753,0.1438$ and 0.10815 Torr using a simple pass cell $(L=65 \mathrm{~cm})$. Experimental points and the Voigt profile fits are represented with circles and straight lines, respectively.
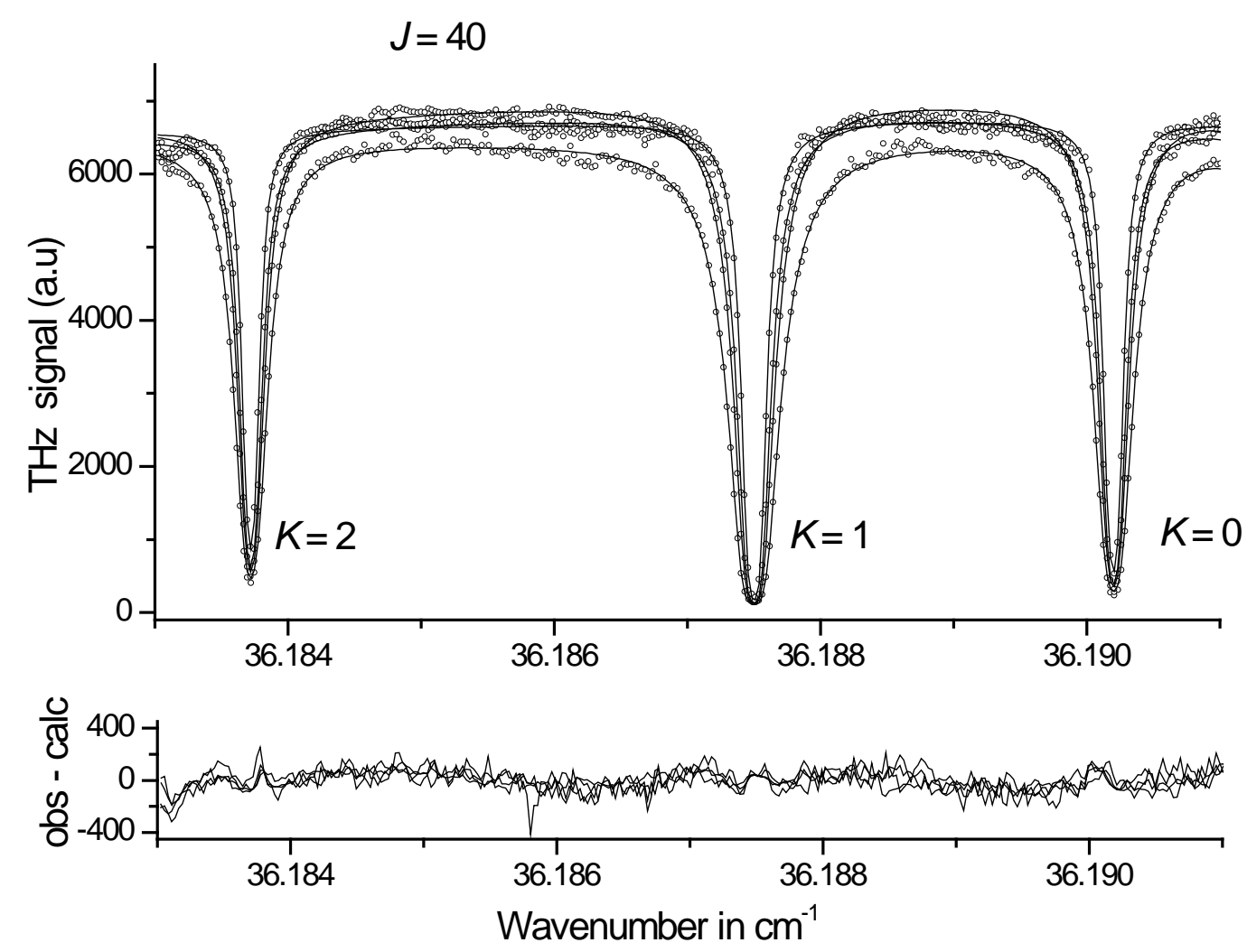


\section{Figure 2:}

$K$-components of the $J=6 \rightarrow 7$ transition for $\mathrm{CH}_{3}{ }^{35} \mathrm{Cl}$ recorded with the frequency-modulation spectrometer at $292 \mathrm{~K}$ with $P=19.9$ mTorr (origin of frequencies is placed at $186.075 \mathrm{GHz}$ ). For the sake of clarity, quantum numbers $K$ are indicated only for most intense lines corresponding to $\Delta F=+1$. The groups of components $K=0-3$ (lower panel) and 4-6 (upper panel) have been fitted separately and have different signal scales. Both signal and residuals (obs.-calc.) are unmagnified and have same scale.
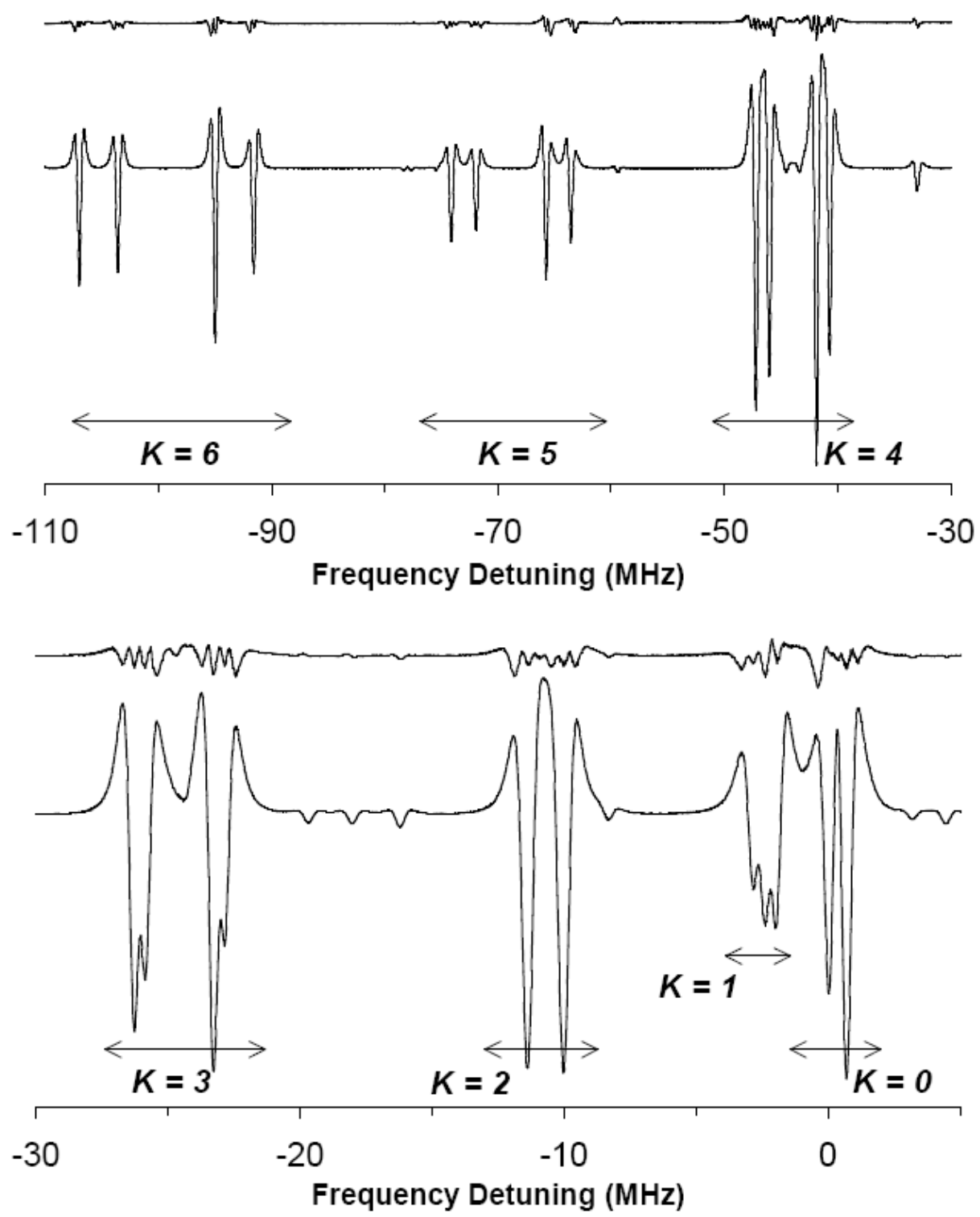


\section{Figure 3:}

Experimental self-broadening coefficients $\gamma_{\text {self }}^{0}$ vs. $K^{2}$ for different $J$ values: FT (solid squares), FM (open diamonds) and PM (open triangles). The straight lines represent the leastsquares linear fits with the quadratic empirical model of Eq. (3). For $J=6$, the straight line corresponds to the linear fit of the FT measurements.
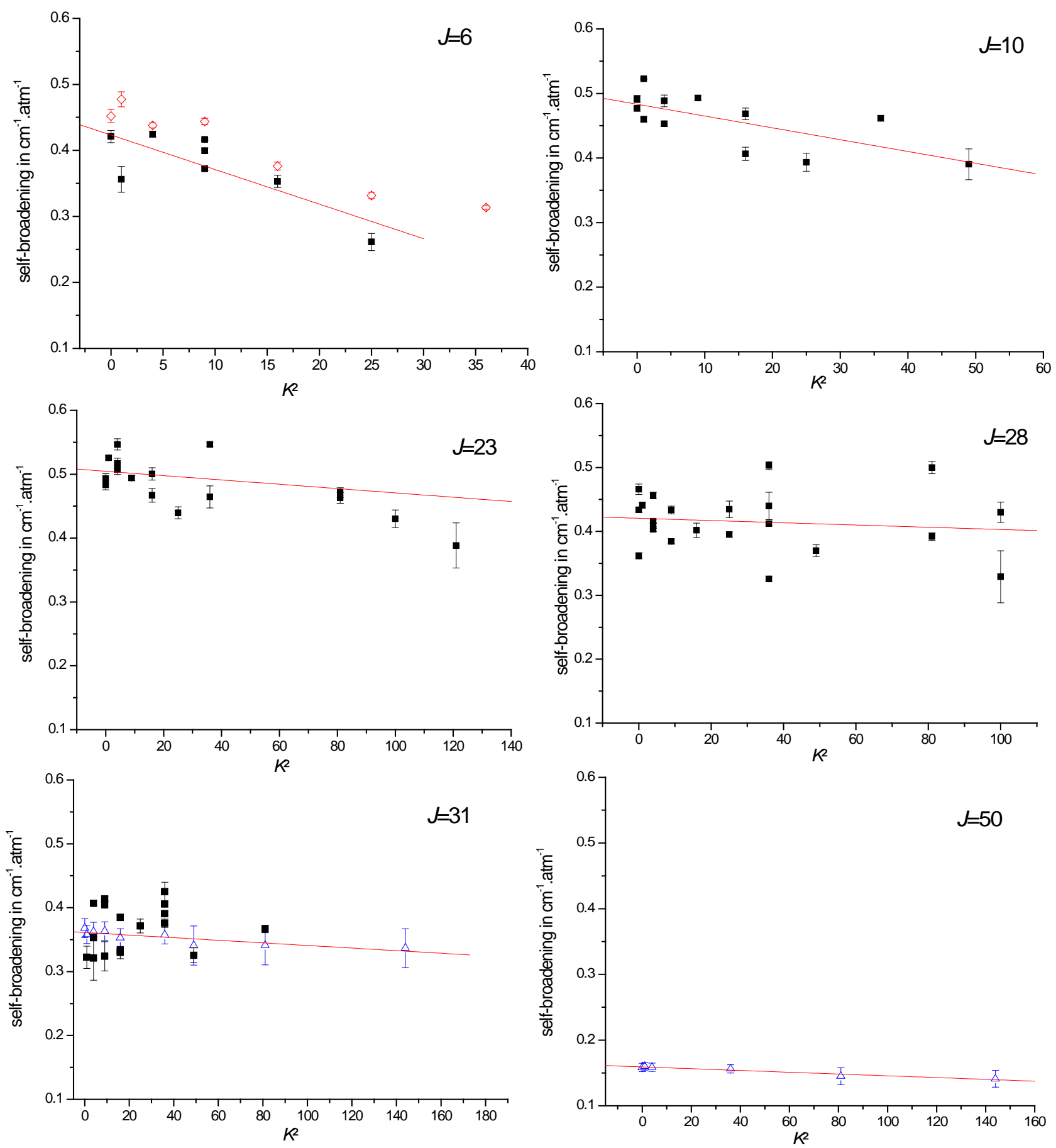


\section{Figure 4:}

Parameters $a_{J}^{0}$ and $a_{J}^{2}$ deduced from the fits (see Eq. (3)) of the various measured selfbroadening coefficients: FTIR (solid squares), FM (open diamond), PM (open triangles). The error bars correspond to one standard deviation. The continuous line represents the smoothed values of these coefficients (see Table 4).
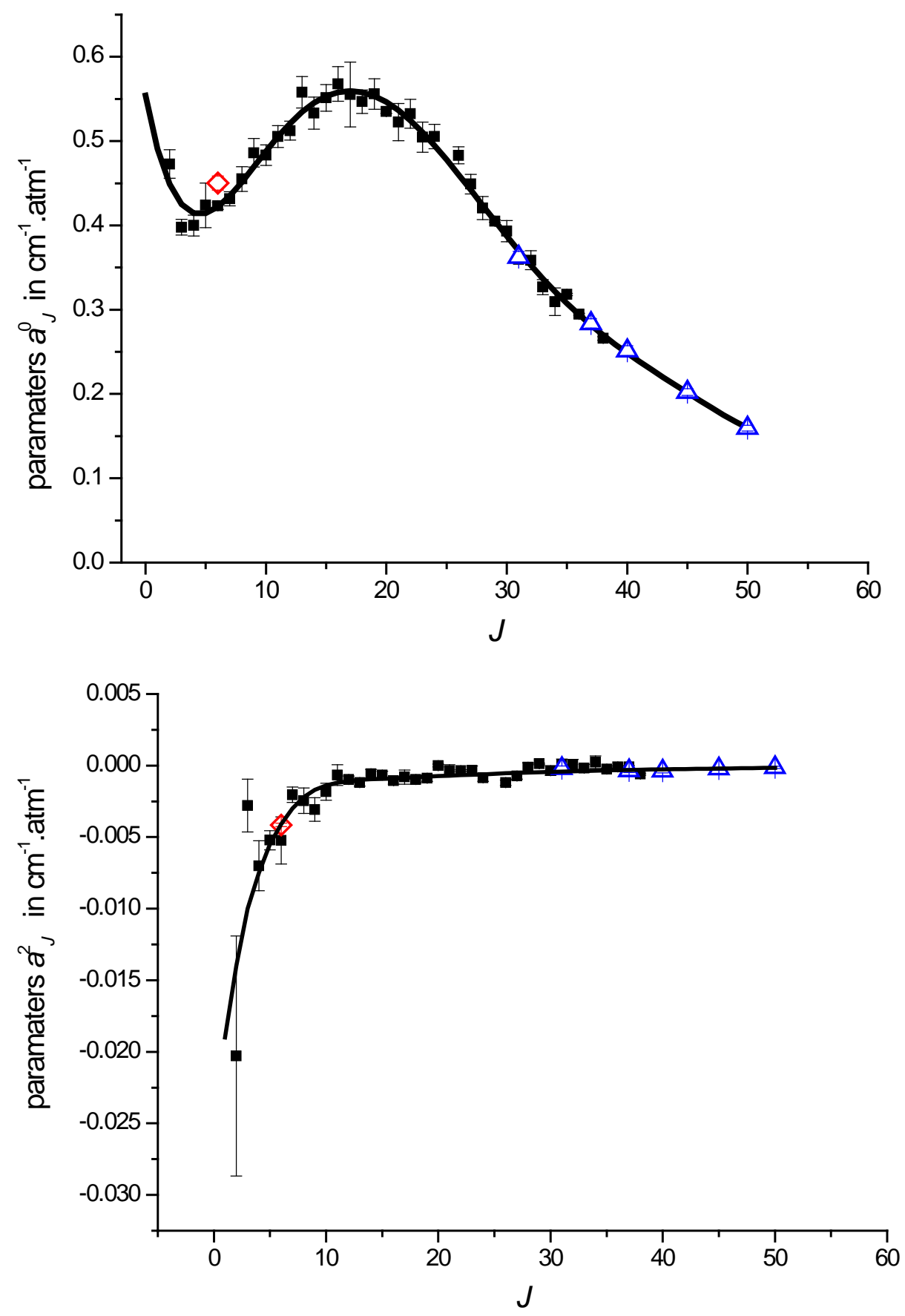


\section{Figure 5:}

$J$ and $K$ dependences observed (FT: solid squares, FM: open diamonds, PM: open triangles) and calculated (empirical model: continuous line, semi-classical calculations with Eq. (4): dashed line) for the self-broadening coefficients obtained in the present work. The $v_{3}$ experimental values of Ref. [7] are also given (solid stars).
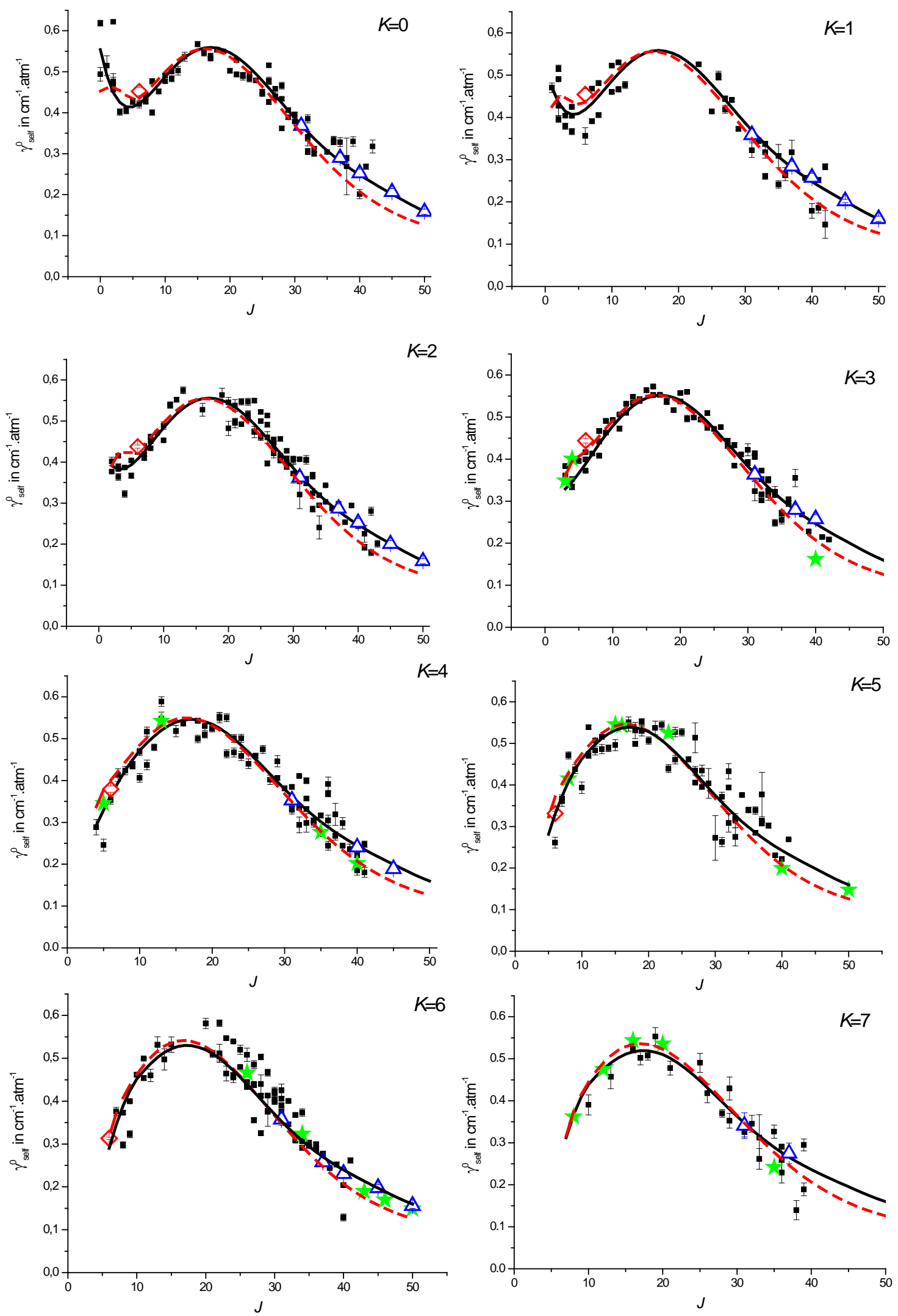


\section{Figure 6:}

Study of convergence of the calculated self-broadening coefficients obtained with Eq. (4) and various electrostatic terms with respect to the maximal intermolecular distance taken into account.

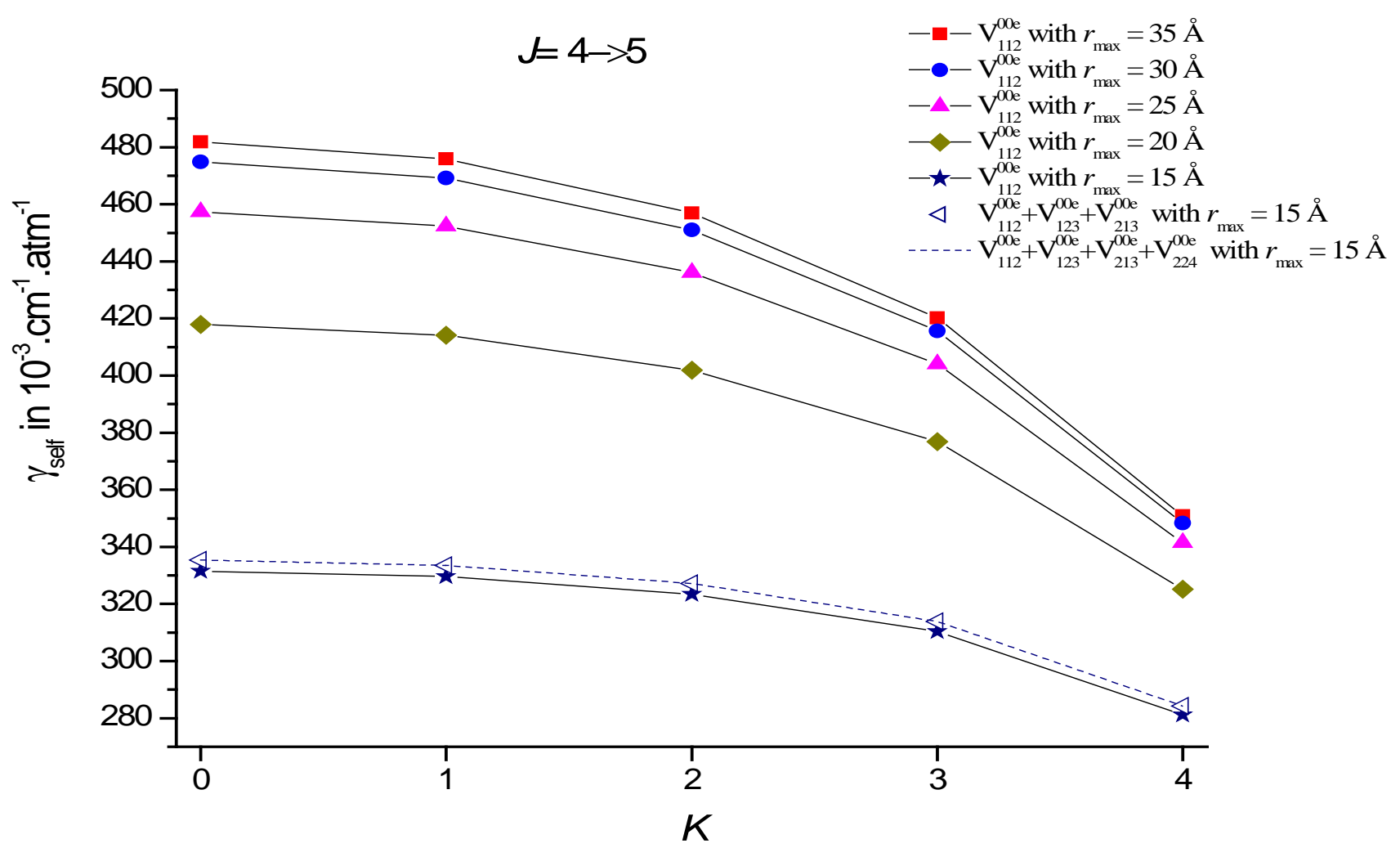




\section{Figure 7:}

Influence of the maximal intermolecular distance accounted for the semi-classical calculations (using Eq. (4)) on the $J$-dependences of self-broadening coefficients for $K=0$ (upper panel) and $K=7$ (lower panel). The results obtained with the electrostatic terms only and with the atom-atom interactions added are practically indistinguishable.
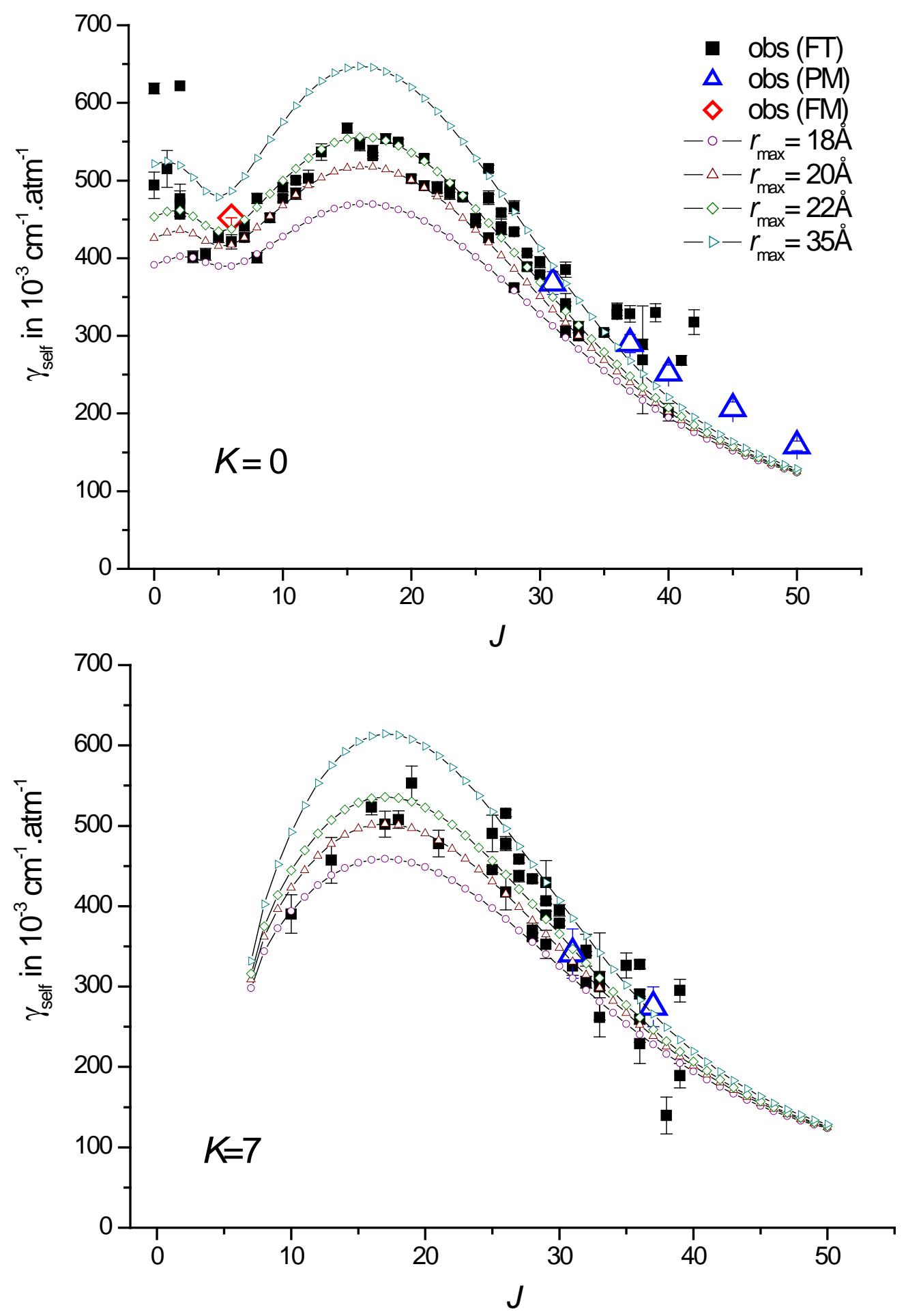


\section{Figure 8:}

Two isotropic potentials studied in the present work: the Lennard-Jones potential with $\varepsilon=368.4 \mathrm{~K}, \sigma=3.584 \AA$ [38] (solid line) and the numerical potential obtained as the isotropic part of the atom-atom interactions (dashed line).

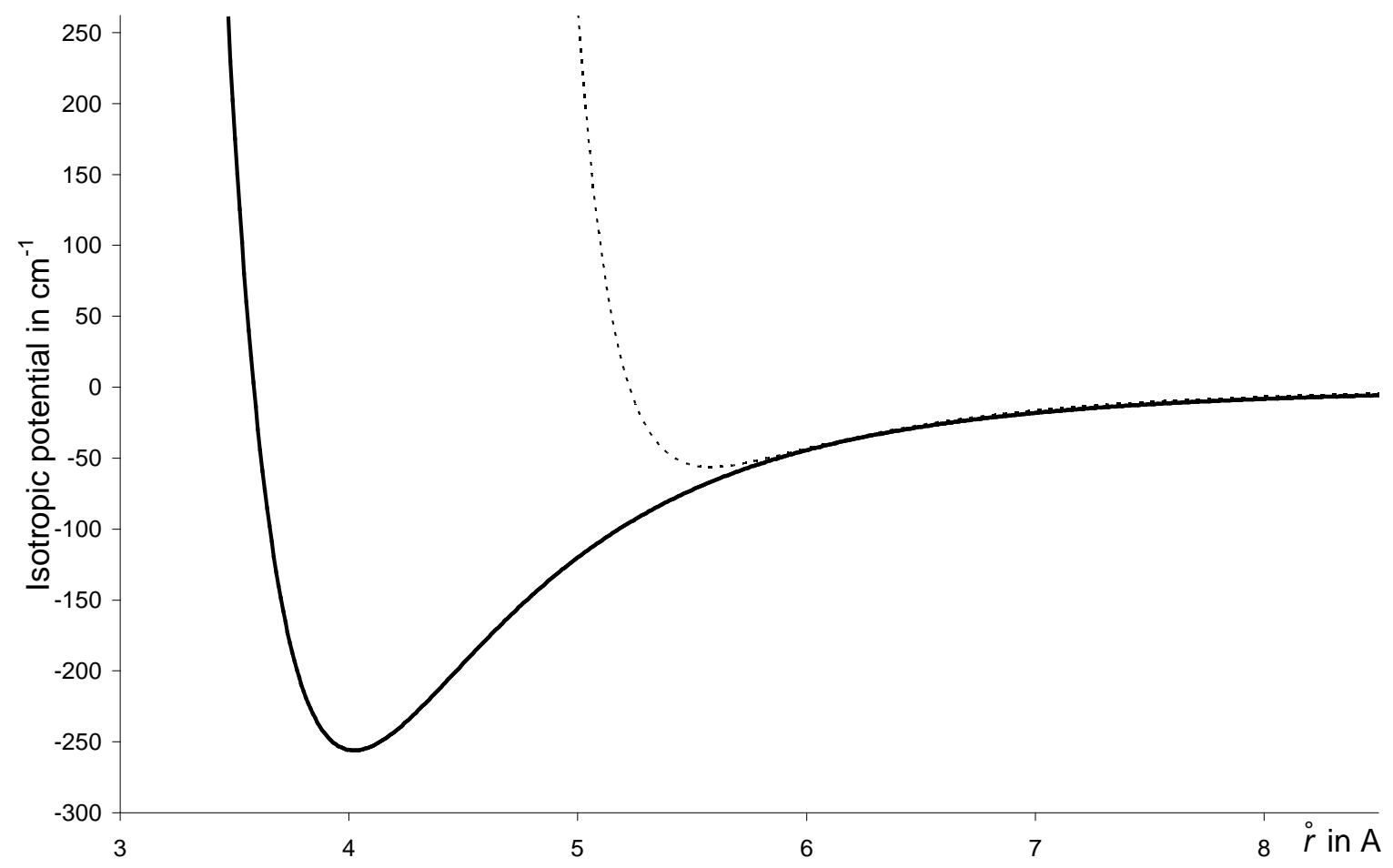




\section{Figure 9:}

Self-broadening coefficients calculated with Eq. (5) for various maximal values of the intermolecular distance for $K=0$ (upper panel) and $K=7$ (lower panel).
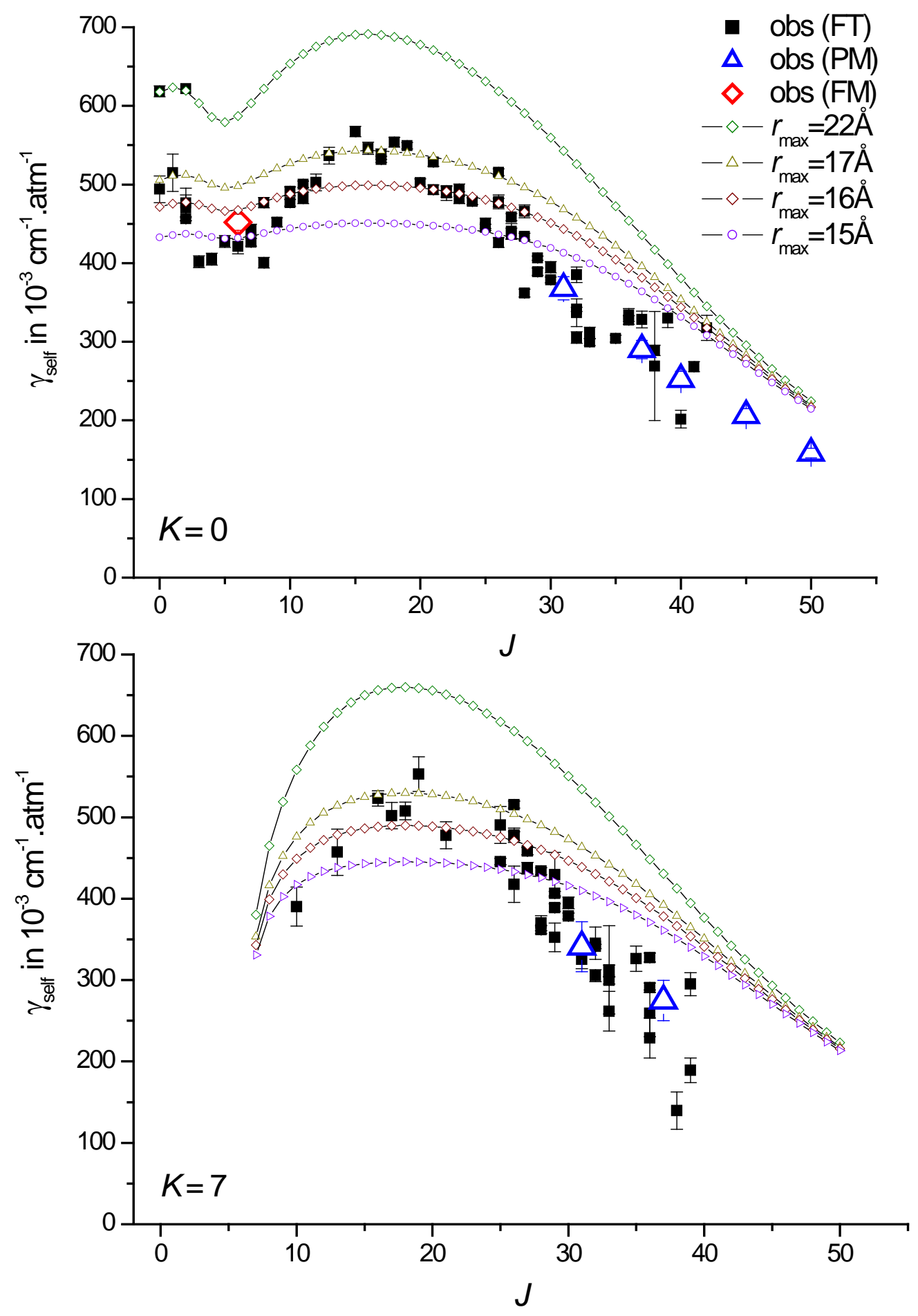


\section{Figure 10:}

Self-broadening coefficients $\gamma_{\text {self }}$ vs. the rotational quantum number $J$ : FT measurements for the isotopologues $\mathrm{CH}_{3}{ }^{35} \mathrm{Cl}$ (solid squares) and $\mathrm{CH}_{3}{ }^{37} \mathrm{Cl}$ (open squares); $\mathrm{FM}$ measurements (open diamonds) and PM measurements (solid triangles) for $\mathrm{CH}_{3}{ }^{35} \mathrm{Cl}$. The continuous line represents the calculations with the polynomial of Ref. [6].

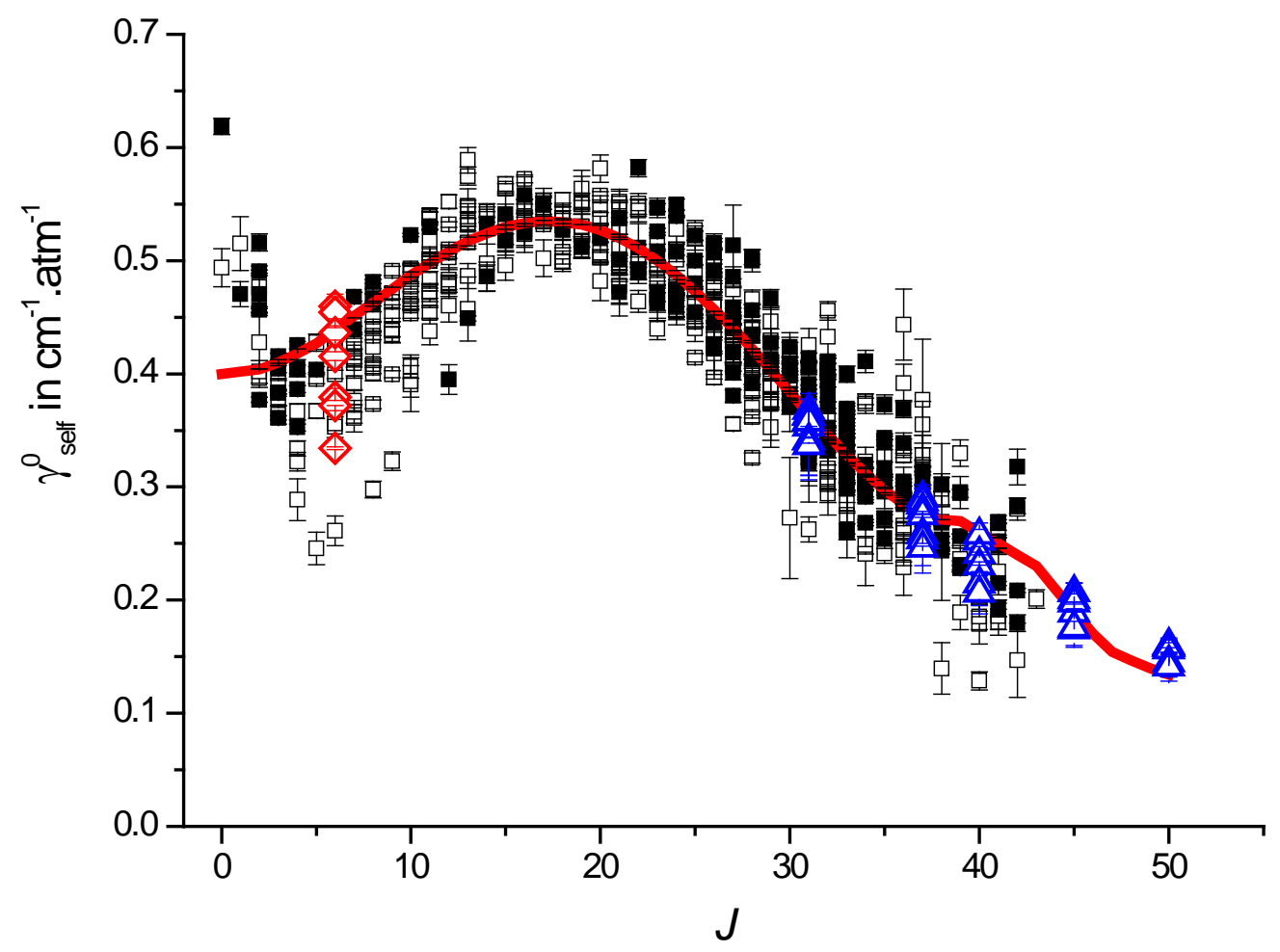

